Supporting Information for:

\title{
Carbon-Nitrogen Bond-Forming Reactions of Palladacycles with Hypervalent Iodine Reagents
}

Allison R. Dick, Matthew S. Remy, Jeff W. Kampf, Melanie S. Sanford*

Department of Chemistry, University of Michigan, 930 North University Avenue, Ann Arbor, MI 48109

\section{Instrumentation}

NMR spectra were obtained on a Varian Inova $500\left(499.90 \mathrm{MHz}\right.$ for ${ }^{1} \mathrm{H} ; 125.70 \mathrm{MHz}$ for $\left.{ }^{13} \mathrm{C}\right)$ or a Varian Inova $400\left(399.96 \mathrm{MHz}\right.$ for ${ }^{1} \mathrm{H} ; 100.57 \mathrm{MHz}$ for $\left.{ }^{13} \mathrm{C}\right)$ spectrometer. ${ }^{1} \mathrm{H}$ NMR chemical shifts are reported in parts per million ( $\mathrm{ppm}$ ) relative to TMS, with the residual solvent peak used as an internal reference. Multiplicities are reported as follows: singlet (s), doublet (d), doublet of doublets (dd), doublet of doublet of doublets (ddd), doublet of triplets (dt), triplet of doublets $(\mathrm{td})$, triplet of triplets $(\mathrm{tt})$, triplet $(\mathrm{t})$, doublet of quartets (dq), quartet (q), multiplet $(\mathrm{m})$, and broad resonance (br). Infrared spectra were obtained on a Perkin-Elmer FT-IR Spectrum BX spectrometer. High resolution mass spectral data were obtained on a Micromass AutoSpec Ultima magnetic sector mass spectrometer.

\section{Materials and Methods}

Benzo[h]quinoline was purchased from TCI America or Aldrich and used as received. Azobenzene was purchased from Acros and recrystallized from ethanol prior to use. $\mathrm{PhI}=\mathrm{NTs}{ }^{1}$ and the OMe- and $\mathrm{NO}_{2}{ }^{-}{ }^{2}$ substituted variants were prepared according to literature procedures from commercially available sulfonamides. Palladium(II) dimers of azobenzene, ${ }^{3}$ benzo[h]quinoline, ${ }^{4}$ and 8 -ethylquinoline ${ }^{5}$ were prepared according to literature procedures. $\mathrm{PdCl}_{2}$ and $\mathrm{Na}_{2} \mathrm{PdCl}_{4}$ were purchased from Pressure Chemical and used as received. Solvents were purchased from Fisher Scientific and used without further purification. All reactions were carried out under ambient atmosphere. Flash chromatography was performed on Silicycle Silica P flash silica gel (40-63 $\mu \mathrm{m}$ particle size, $60 \AA$ pore diameter, $50 \mathrm{~m}^{2} / \mathrm{g}$ surface area) and thin layer chromatography was performed on EMD TLC plates pre-coated with $250 \mu \mathrm{m}$ thickness silica gel $60 \mathrm{~F}_{254}$. Preparative TLC was performed on Whatman PK6F silica gel plates (60 $\AA, 500$ $\mu \mathrm{m}$ layer thickness).

\section{Additional Experimental Details}

\section{$\underline{\text { Reaction of PhI=NTs with Azobenzene Complex } 1}$}

Product 3: Procedure to determine yield by ${ }^{1} H$ NMR spectroscopy: Complex 1 (50.0 mg, 0.077

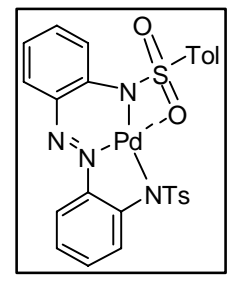
mmol, 1 equiv) was dissolved in $\mathrm{MeCN}(5.0 \mathrm{~mL})$, and PhINTs $(1,2$, or 4 equiv relative to [Pd]) was added. The resulting suspension was stirred at $25^{\circ} \mathrm{C}$ for $12 \mathrm{~h}$ during which time the color changed bright orange to dark purple. Unreacted starting material (a yellow solid) was removed by filtration, and the remaining solution was evaporated to dryness. The solids were dissolved in $\mathrm{CDCl}_{3}, 1,3,5-$ 
trimethoxybenzene was added as an internal standard, and reaction yield was determined by ${ }^{1} \mathrm{H}$ NMR spectroscopy. The yield of 3 was determined to be $19 \%$ (with 1 equiv of $\mathrm{PhI}=\mathrm{NTs}$ ), $32 \%$ (with 2 equiv $\mathrm{PhI}=\mathrm{NTs}$ ), and $50 \%$ (with 4 equiv of $\mathrm{PhI}=\mathrm{NTs}$ ). The procedure for isolation of complex $\mathbf{3}$ is described in detail in the manuscript.

Notes about ${ }^{1} \mathrm{H}$ NMR characterization data of 3 . The ${ }^{1} \mathrm{H}$ NMR signals associated with the Ts groups shifted significantly depending on concentration. In addition, the ${ }^{1} \mathrm{H}$ NMR spectra of $\mathbf{3}$ often contained broad singlets, with chemical shifts that varied dramatically with concentration (see spectra on pages S2 and S3 of the other SI file for examples). We hypothesized that these signals were due to coordinated/associated water molecules, and this was confirmed using the following procedure: A ${ }^{1} \mathrm{H}$ NMR spectrum of $\mathbf{3}$ was obtained, and shown to contain a broad singlet at $5.35 \mathrm{ppm}$. Pyridine (2 equiv) was added directly to the NMR tube, and the ${ }^{1} \mathrm{H}$ NMR spectrum was reacquired. Upon the addition of pyridine, new signals associated with both free and Pd-bound pyridine appeared in the aromatic region of the ${ }^{1} \mathrm{H}$ NMR spectrum. The peak at $5.35 \mathrm{ppm}$ was no longer present, and a new peak appeared at $1.59 \mathrm{ppm}$, which was consistent with free $\mathrm{H}_{2} \mathrm{O}$.

\section{Reaction of PhI=NTs with benzo[h]quinoline complexes 4,5,6 and 7.}

Products 8a and 9a: Procedure to determine yield by 1H NMR spectroscopy: Complex 4 (12.5 $\mathrm{mg}, 0.02 \mathrm{mmol}, 1$ equiv) and $\mathrm{PhI}=\mathrm{NTs}(17.6 \mathrm{mg}, 0.05 \mathrm{mmol}, 1.3$ equiv relative to [Pd]) were weighed into six $4 \mathrm{~mL}$ vials. Each vial was equipped with a Teflon stirbar, and $\mathrm{MeCN}(2 \mathrm{~mL})$ was added to each vial. Pyridine- $d_{5}(25 \mu \mathrm{L}, 0.33 \mathrm{mmol}, 36$ equiv relative to $[\mathrm{Pd}])$ was immediately added to three of the vials to generate complex 6 in situ, and then all reactions were stirred overnight. The solvent was removed under vacuum, and each resulting oil was dissolved in $0.7 \mathrm{~mL}$ of a $3: 1 \mathrm{CDCl}_{3}: \mathrm{C}_{5} \mathrm{D}_{5} \mathrm{~N}$ solution containing 1,3,5-trimethoxybenzene as an internal standard. Yields of 8a and 9a were determined by ${ }^{1} \mathrm{H}$ NMR, and average yields were calculated for reactions from $\mathbf{4}$ (three vials without added pyridine) and $\mathbf{6}$ (three vials with added pyridine).

Products 8b and 9b: Procedure to determine yield by ${ }^{1} H$ NMR spectroscopy: The same procedure was followed as that used for determination of 8a and 9a yields, only complex $\mathbf{5}$ (11.6 $\mathrm{mg}, 0.02 \mathrm{mmol}, 1$ equiv) was used instead of complex 4 .

\section{Acid Cleavage Reactions}

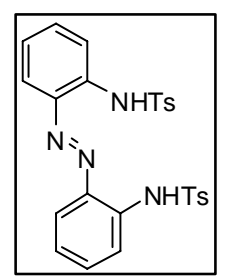

HCl cleavage of 3. Complex 3 ( $29.3 \mathrm{mg}, 0.047 \mathrm{mmol}, 1$ equiv) was dissolved in $\mathrm{MeCN}(10 \mathrm{~mL})$, and $1 \mathrm{M} \mathrm{HCl}$ in ether $(0.465 \mathrm{~mL}, 0.47 \mathrm{mmol}, 10$ equiv) was added. The purple solution immediately changed color to orange. The reaction was stirred for $5 \mathrm{~h}$, during which time an orange solid precipitated from solution. The solid was collected on a fritted filter, washed with $\mathrm{MeCN}(10 \mathrm{~mL})$, and dried under vacuum. The product ( $\left.3^{\prime}\right)$ was obtained as an orange solid $(18.4 \mathrm{mg}, 75 \%$ yield). Note: this reaction proceeded to $100 \%$ conversion as determined by ${ }^{1} \mathrm{H}$ NMR spectroscopy. ${ }^{1} \mathrm{H}$ NMR (acetone- $\left.d_{6}\right): \delta 7.72(2 \mathrm{H}, \mathrm{dd}, J=8.5 \mathrm{~Hz}, 1.0 \mathrm{~Hz}), 7.66(4 \mathrm{H}, \mathrm{d}, J=8.0 \mathrm{~Hz}), 7.57(2 \mathrm{H}, \mathrm{dd}, J$ $=8.0 \mathrm{~Hz}, 1.5 \mathrm{~Hz}), 7.53(2 \mathrm{H}, \mathrm{ddd}, J=8.5 \mathrm{~Hz}, 7.5 \mathrm{~Hz}, 1.5 \mathrm{~Hz}), 7.23(2 \mathrm{H}, \mathrm{ddd}, J=8.0 \mathrm{~Hz}, 7.0 \mathrm{~Hz}$, 
$1.0 \mathrm{~Hz}), 7.19(4 \mathrm{H}, \mathrm{d}, J=8.0 \mathrm{~Hz}), 2.80(2 \mathrm{H}, \mathrm{s}), 2.24(6 \mathrm{H}, \mathrm{s}) .{ }^{13} \mathrm{C}$ NMR (DMSO-d $\left.)_{6}\right): \delta 143.68$, $143.09,136.93,136.10,132.38,129.37,126.57,125.24,124.61,117.24,20.80$. HRMSelectrospray $(\mathrm{m} / \mathrm{z}):[\mathrm{M}+\mathrm{H}]^{+}$calcd for $\mathrm{C}_{26} \mathrm{H}_{25} \mathrm{~N}_{4} \mathrm{O}_{4} \mathrm{~S}_{2}: 521.1317$; found: 521.1321 .

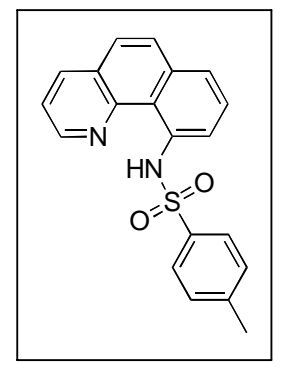

HCl Cleavage of $\mathbf{8 b}$. Complex $\mathbf{8 b}(61.0 \mathrm{mg}, 0.11 \mathrm{mmol}, 1$ equiv) was weighed into a $20 \mathrm{~mL}$ scintillation vial equipped with a Teflon stirbar. MeCN $(8 \mathrm{~mL})$ and $\mathrm{HCl}$ (as a $1 \mathrm{M}$ solution in $\mathrm{Et}_{2} \mathrm{O}, 5$ equiv) were added. The reaction was stirred for $24 \mathrm{~h}$, and then diluted with saturated $\mathrm{NaHCO}_{3}(50 \mathrm{~mL})$. The aqueous layer was extracted with ethyl acetate $(3 \times 50 \mathrm{~mL})$, and the combined organic layers were dried with $\mathrm{MgSO}_{4}$, filtered, and evaporated to dryness. The resulting solid was purified by chromatography on silica gel $\left(\mathrm{R}_{\mathrm{f}}=0.14\right.$ in $80 \%$ hexanes, $20 \%$ ethyl acetate). The product $\mathbf{8} \mathbf{b}^{\prime}$ was isolated as a pale yellow solid ( $12.1 \mathrm{mg}, 32 \%$ yield). Note: this reaction proceeded to $100 \%$ conversion as determined by ${ }^{1} \mathrm{H}$ NMR spectroscopy. ${ }^{1} \mathrm{H}$ NMR $\left(\mathrm{CDCl}_{3}\right): \delta 8.98(1 \mathrm{H}, \mathrm{dd}, J=4.5 \mathrm{~Hz}, 1.4 \mathrm{~Hz}), 8.25(1 \mathrm{H}, \mathrm{dd}, J=$ $8.0 \mathrm{~Hz}, 1.6 \mathrm{~Hz}), 7.91(1 \mathrm{H}, \mathrm{dd}, J=7.4 \mathrm{~Hz}, 1.3 \mathrm{~Hz}), 7.80(2 \mathrm{H}, \mathrm{d}, J=8.25 \mathrm{~Hz}), 7.74(1 \mathrm{H}, \mathrm{d}, J=8.8$ $\mathrm{Hz}), 7.63(1 \mathrm{H}, \mathrm{d}, J=8.8 \mathrm{~Hz}), 7.61-7.52(3 \mathrm{H}$, multiple peaks), $7.09(2 \mathrm{H}, \mathrm{d}, J=8.5 \mathrm{~Hz}), 2.23(3 \mathrm{H}$, s), $-0.72(1 \mathrm{H}, \mathrm{br} \mathrm{s}) .{ }^{1} \mathrm{H}$ NMR $\left(\mathrm{C}_{6} \mathrm{D}_{6}\right): \delta 8.39(1 \mathrm{H}, \mathrm{dd}, J=8.0 \mathrm{~Hz}, 1.0 \mathrm{~Hz}), 8.33(1 \mathrm{H}, \mathrm{dd}, J=4.5$ $\mathrm{Hz}, 1.8 \mathrm{~Hz}), 7.96(2 \mathrm{H}, \mathrm{dt}, J=8.5 \mathrm{~Hz}, 1.7 \mathrm{~Hz}), 7.33(1 \mathrm{H}, \mathrm{dd}, J=8.0 \mathrm{~Hz}, 1.8 \mathrm{~Hz}), 7.27(1 \mathrm{H}, \mathrm{t}, J=$ $7.9 \mathrm{~Hz}), 7.21(1 \mathrm{H}, \mathrm{d}, J=8.8 \mathrm{~Hz}), 7.12(1 \mathrm{H}, \mathrm{dd}, J=7.9 \mathrm{~Hz}, 0.6), 6.95(1 \mathrm{H}, \mathrm{d}, J=8.8 \mathrm{~Hz}), 6.68$ $(1 \mathrm{H}, \mathrm{dd}, J=8.8 \mathrm{~Hz}, 4.5 \mathrm{~Hz}), 6.49(2 \mathrm{H}, \mathrm{d}, J=8.0 \mathrm{~Hz}), 1.59(3 \mathrm{H}, \mathrm{s}),-0.49(1 \mathrm{H}, \mathrm{br} \mathrm{s}) .{ }^{13} \mathrm{C}$ NMR $\left(\mathrm{CDCl}_{3}\right): \delta 147.56,145.97,143.27,138.73,137.41,136.85,135.29,129.52,129.24,128.80$, $127.43,127.37,125.51,122.89,121.32,117.49,116.28,21.55$. HRMS-electrospray $(\mathrm{m} / \mathrm{z})$ : $[\mathrm{M}+\mathrm{H}]^{+}$calcd for $\mathrm{C}_{20} \mathrm{H}_{17} \mathrm{~N}_{2} \mathrm{O}_{2} \mathrm{~S}, 349.1011$; found, 349.1018.

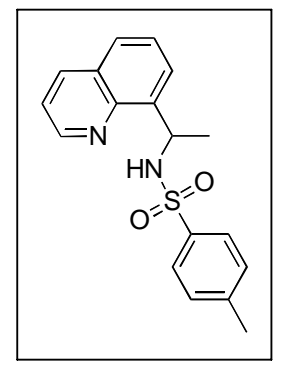

HCl Cleavage of 12. Complex 12 (34.7 mg, $0.064 \mathrm{mmol}, 1$ equiv) was weighed into a $20 \mathrm{~mL}$ scintillation vial equipped with a Teflon stirbar. $\mathrm{MeCN}$ $(8 \mathrm{~mL})$ and $\mathrm{HCl}$ (as a $1 \mathrm{M}$ solution in $\mathrm{Et}_{2} \mathrm{O}$ ) were added. The reaction was stirred for $24 \mathrm{~h}$, and then diluted with saturated $\mathrm{NaHCO}_{3}(50 \mathrm{~mL})$. The aqueous layer was extracted with ethyl acetate $(3 \times 50 \mathrm{~mL})$, and the combined organic layers were dried with $\mathrm{MgSO}_{4}$, filtered, and evaporated to dryness. Analysis of the crude reaction mixture by ${ }^{1} \mathrm{H}$ NMR spectroscopy showed multiple aromatic products. The crude mixture was purified by chromatography on silica gel $\left(\mathrm{R}_{\mathrm{f}}\right.$ $=0.12$ in $80 \%$ hexanes, $20 \%$ ethyl acetate), and the product (12') was isolated as a white solid (8.6 mg, $42 \%$ yield). ${ }^{1} \mathrm{H}$ NMR $\left(\mathrm{CDCl}_{3}\right): \delta 8.78(1 \mathrm{H}, \mathrm{dd}, J=4.2 \mathrm{~Hz}, 1.8 \mathrm{~Hz}), 8.02(1 \mathrm{H}, \mathrm{dd}, J=$ $8.3 \mathrm{~Hz}, 1.8 \mathrm{~Hz}), 7.62(1 \mathrm{H}, \mathrm{br} \mathrm{d}, \mathrm{J}=9.9 \mathrm{~Hz}), 7.53(1 \mathrm{H}, \mathrm{dd}, J=7.5 \mathrm{~Hz}, 2.1 \mathrm{~Hz}), 7.36(1 \mathrm{H}, \mathrm{dd}, J=$ $8.3 \mathrm{~Hz}, 4.2 \mathrm{~Hz}), 7.30-7.22(4 \mathrm{H}$, multiple peaks), $6.67(2 \mathrm{H}, \mathrm{d}, J=7.9 \mathrm{~Hz}), 4.86(1 \mathrm{H}, \mathrm{dq}, J=9.9$ $\mathrm{Hz}, 7.1 \mathrm{~Hz}), 2.12(3 \mathrm{H}, \mathrm{s}), 1.70(3 \mathrm{H}, \mathrm{d}, J=7.1 \mathrm{~Hz}) .{ }^{1} \mathrm{H}$ NMR $\left(\mathrm{C}_{6} \mathrm{D}_{6}\right): \delta 8.24(1 \mathrm{H}, \mathrm{dd}, J=4.2 \mathrm{~Hz}$, $1.8 \mathrm{~Hz}), 7.53(1 \mathrm{H}, \mathrm{br} \mathrm{d}, J=9.7 \mathrm{~Hz}), 7.45(2 \mathrm{H}, \mathrm{dt}, J=8.4 \mathrm{~Hz}, 1.9 \mathrm{~Hz}), 7.27(1 \mathrm{H}, \mathrm{dd}, J=8.3 \mathrm{~Hz}$, $1.8 \mathrm{~Hz}), 7.00(1 \mathrm{H}, \mathrm{dd}, J=8.1 \mathrm{~Hz}, 1.5 \mathrm{~Hz}), 6.92(2 \mathrm{H}, \mathrm{dd}, J=7.0 \mathrm{~Hz}, 1.4 \mathrm{~Hz}), 6.83(1 \mathrm{H}, \mathrm{dd}, J=$ $7.1 \mathrm{~Hz}, 1.0 \mathrm{~Hz}), 6.58(1 \mathrm{H}, \mathrm{dd}, J=8.3 \mathrm{~Hz}, 4.2 \mathrm{~Hz}), 6.30(2 \mathrm{H}, \mathrm{d}, J=8.0 \mathrm{~Hz}), 5.12(1 \mathrm{H}, \mathrm{dq}, J=9.9$ $\mathrm{Hz}, 7.1 \mathrm{~Hz}), 1.67(3 \mathrm{H}, \mathrm{s}), 1.61(3 \mathrm{H}, \mathrm{d}, J=7.0 \mathrm{~Hz}) .{ }^{13} \mathrm{C} \mathrm{NMR}\left(\mathrm{CDCl}_{3}\right): \delta 148.7,145.5,142.0$, $138.4,137.5,136.8,128.9,128.6,128.4,127.4,126.5,126.2,121.1,56.0,24.4,21.3$. HRMSelectrospray $(\mathrm{m} / \mathrm{z}):[\mathrm{M}+\mathrm{H}]^{+}$calcd for $\mathrm{C}_{18} \mathrm{H}_{19} \mathrm{~N}_{2} \mathrm{O}_{2} \mathrm{~S}, 327.1167$; found, 327.1152 . 


\section{$\underline{\text { Characterization of Organic Side Products }}$}

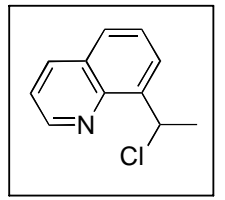

Product 13: This product was observed by ${ }^{1} \mathrm{H}$ NMR spectroscopy in reactions of 10 with $\mathrm{PhI}=\mathrm{NTs}$ in the absence of AgOTf. The identity of $\mathbf{1 2}$ was confirmed by preparation of an authentic sample via Pd-catalyzed chlorination of 8ethylquinoline with $\mathrm{CuCl}_{2}$. ${ }^{4}$ Product 10 was purified by chromatography on silica gel $\left(\mathrm{R}_{\mathrm{f}}=0.15\right.$ in $95 \%$ hexanes, $5 \%$ ethyl acetate $)$ and was isolated as a white solid (41.2 mg, 9\% yield). ${ }^{1} \mathrm{H} \mathrm{NMR}\left(\mathrm{CDCl}_{3}\right): \delta 8.96(1 \mathrm{H}, \mathrm{dd}, J=4.2 \mathrm{~Hz}, 1.8 \mathrm{~Hz}), 8.17$ $(1 \mathrm{H}, \mathrm{dd}, J=8.3 \mathrm{~Hz}, 1.8 \mathrm{~Hz}), 8.02(1 \mathrm{H}, \mathrm{dd}, J=7.3 \mathrm{~Hz}, 1.4 \mathrm{~Hz}), 7.78(1 \mathrm{H}, \mathrm{dd}, J=8.3 \mathrm{~Hz}, 1.4 \mathrm{~Hz})$, 7.59, (1H, dd, $J=7.8 \mathrm{~Hz}, 7.6 \mathrm{~Hz}), 7.43(1 \mathrm{H}, \mathrm{dd}, J=8.3 \mathrm{~Hz}, 4.2 \mathrm{~Hz}), 6.66(1 \mathrm{H}, \mathrm{q}, J=6.8 \mathrm{~Hz})$, $1.99(3 \mathrm{H}, \mathrm{d}, J=6.9 \mathrm{~Hz}) .{ }^{13} \mathrm{C} \mathrm{NMR}\left(\mathrm{CDCl}_{3}\right): \delta 150.0,144.9,141.1,136.5,128.3,128.1,127.1$, 126.6, 121.4, 53.3, 26.5. HRMS-electrospray $(\mathrm{m} / \mathrm{z}): \mathrm{M}^{+}$calcd for $\mathrm{C}_{11} \mathrm{H}_{10} \mathrm{ClN}, 191.0502$; found, 191.0495 .

Kinetic Measurements for Reaction of Differentially Substituted Iminoiodinane reagents with benzo[h]quinoline monomer (7)

Complex 7 (3.0 mg, $0.0075 \mathrm{mmol}, 1$ equiv) and the iminoiodinane reagent $(0.03 \mathrm{mmol}, 4.0$ equiv) were weighed into each of 24 vials. $\mathrm{CD}_{3} \mathrm{CN}(0.6 \mathrm{~mL})$ was added, and the reactions were stirred at $25{ }^{\circ} \mathrm{C}$ for various time intervals. The contents of each vial were transferred to an NMR tube, and a ${ }^{1} \mathrm{H}$ NMR spectrum was recorded immediately.

Percent Conversion vs. Time For Amination of $\mathbf{7}$ with Differentially Substituted Iminoiodinanes

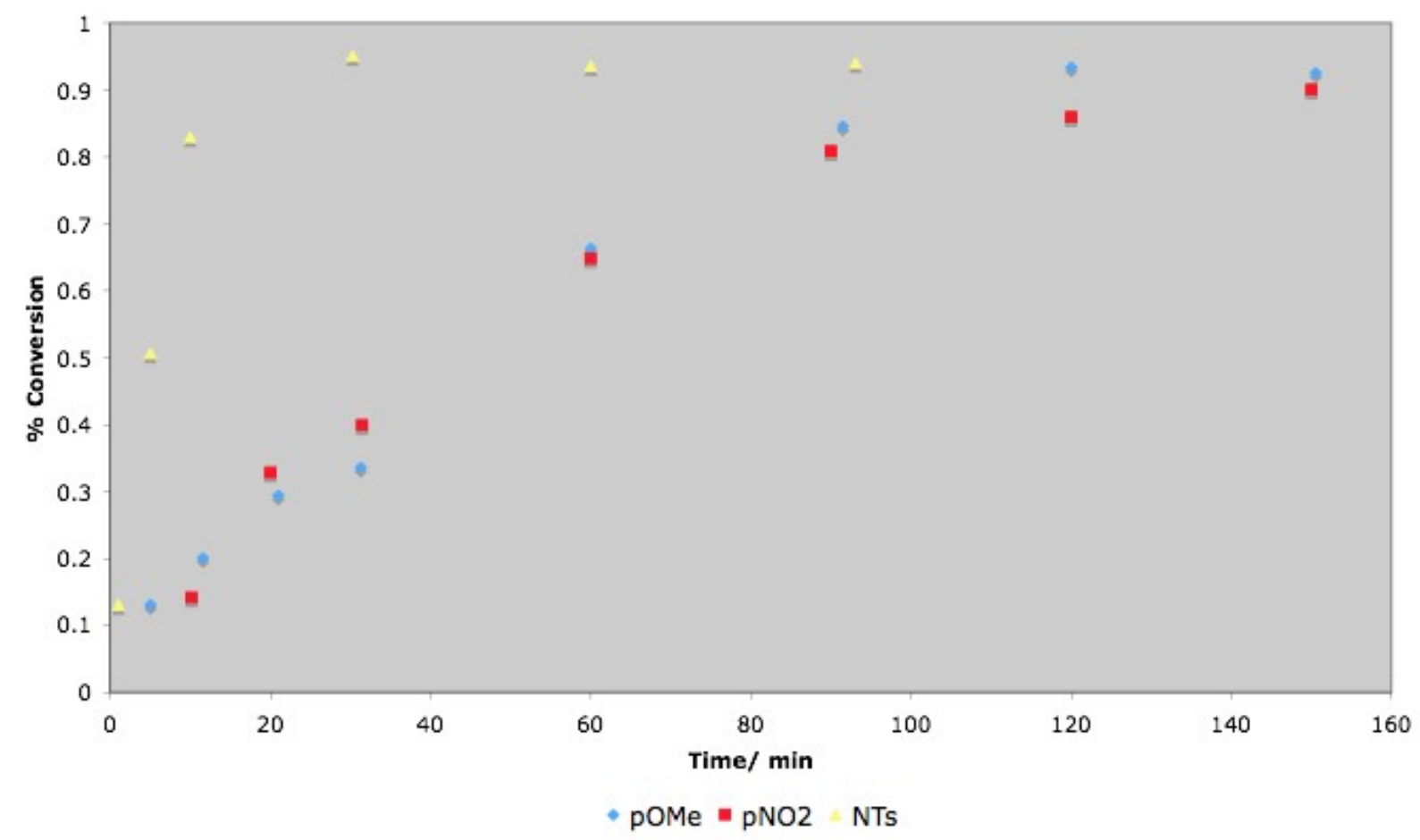




\section{Crystallographic Details}

\section{For azobenzene complex 3:}

Crystal Size: 0.16 x $0.01 \times 0.007 \mathrm{~mm}$

Dark red needle

Mounted in a loop with paratone-N

Intensity data were collected at $193 \mathrm{~K}$ on a Kappa goniostat equipped with a Bruker Platimum 200 CCD detector at Beamline 11.3.1 at the Advanced Light Source (Lawrence Berkeley National Laboratory) using synchrotron radiation tuned to $\lambda=0.77580 \Delta$. The detector was set at a distance of $7.4 \mathrm{~cm}$ from the crystal. A series of data frames measured at $0.2^{\circ}$ increments of $\omega$ were collected to calculate a unit cell. For data collection frames were measured for a duration of 5-s at $0.3^{\circ}$ intervals of $\omega$. The data frames were collected using the program APEX2 and processed using the program SAINT routine within APEX2. The data were corrected for absorption and beam corrections based on the multi-scan technique as implemented in SADABS.

- Crystallographic data were collected through the SCrALS (Service Crystallography at Advanced Light Source) program at the Small-Crystal Crystallography Beamline 11.3.1 at the Advanced Light Source (ALS). The ALS is supported by the U.S. Department of Energy, Office of Energy Sciences Materials Sciences Division, under contract DE-AC03-76SF00098 at Lawrence Berkeley National Laboratory.

- APEX2 v2.0-2 and SAINT v7.23A data collection and data processing programs, respectively. Bruker Analytical X-ray Instruments, Inc., Madison, WI; SADABS v2.10 semiempirical absorption and beam correction program. G.M. Sheldrick, University of Göttingen, Germany;
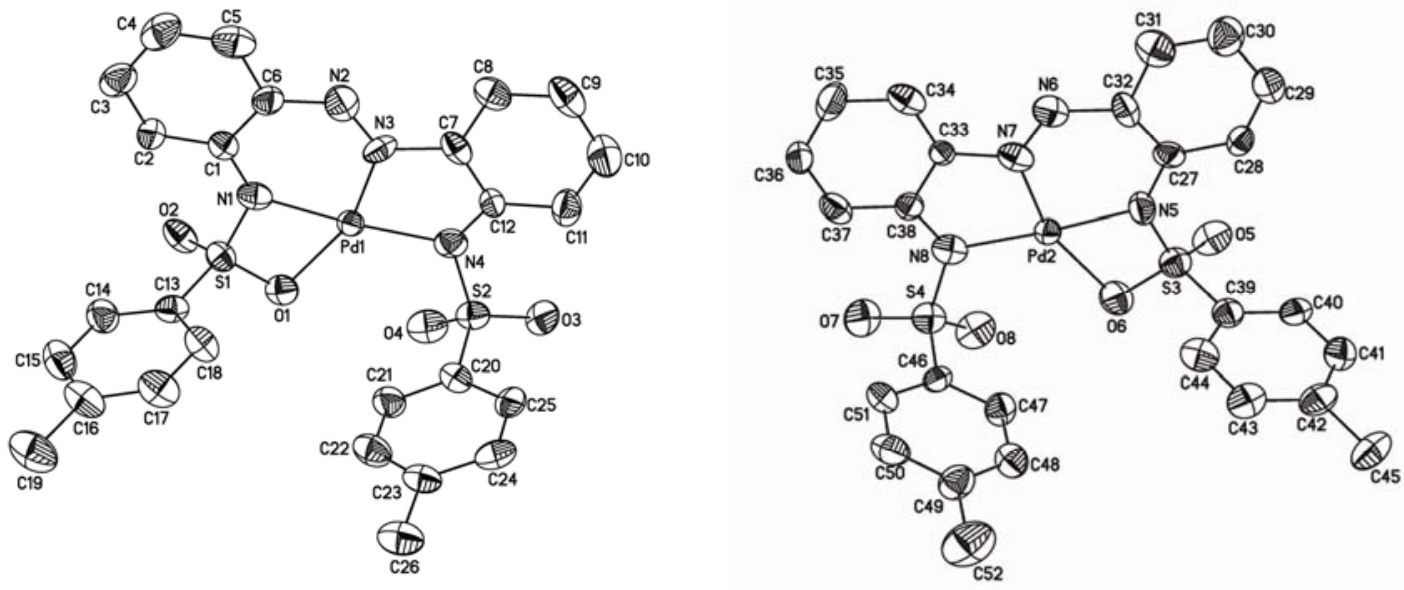

Table 1. Crystal data and structure refinement for ad96a. 


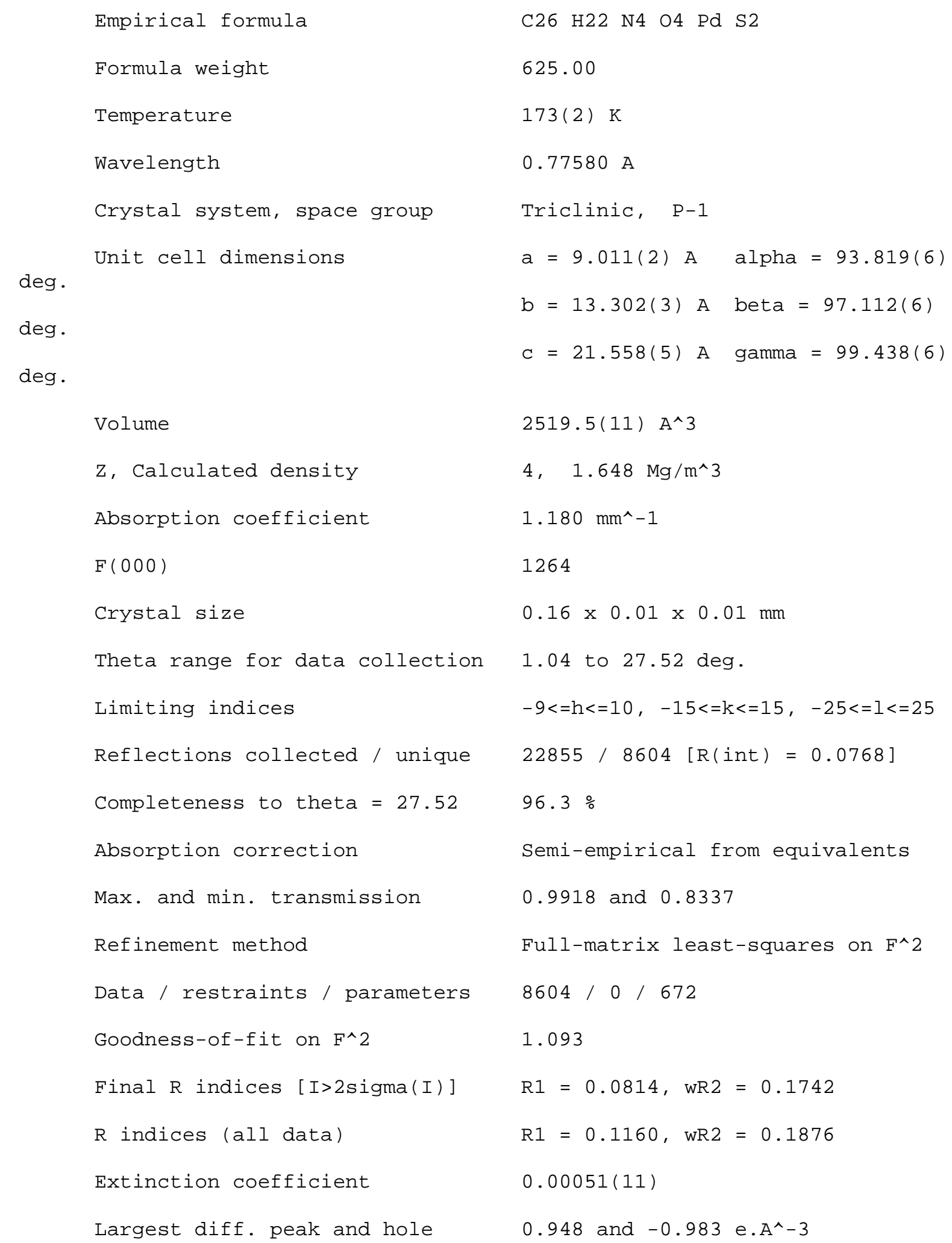

Table 2. Atomic coordinates $(x 10 \wedge 4)$ and equivalent isotropic 
displacement parameters $\left(A^{\wedge} 2 \times 10^{\wedge} 3\right)$ for ad96a.

$U(e q)$ is defined as one third of the trace of the orthogonalized Uij tensor.

\begin{tabular}{|c|c|c|c|c|}
\hline & $x$ & $y$ & $z$ & $\mathrm{U}(\mathrm{eq})$ \\
\hline $\operatorname{Pd}(1)$ & $1759(1)$ & 1388(1) & $4748(1)$ & $28(1)$ \\
\hline $\operatorname{Pd}(2)$ & $7161(1)$ & $1538(1)$ & 181(1) & $31(1)$ \\
\hline$S(1)$ & $2645(3)$ & $2075(2)$ & $3629(1)$ & $36(1)$ \\
\hline$s(2)$ & $648(3)$ & $2803(2)$ & $5758(1)$ & $38(1)$ \\
\hline$s(3)$ & $8968(3)$ & $2651(2)$ & $1240(1)$ & $42(1)$ \\
\hline$s(4)$ & $5168(3)$ & $2526(2)$ & $-820(1)$ & $42(1)$ \\
\hline $0(1)$ & $1877(7)$ & $2631(5)$ & $4068(3)$ & $40(2)$ \\
\hline $0(2)$ & $1995(8)$ & $1945(5)$ & $2984(3)$ & $48(2)$ \\
\hline $0(3)$ & $-239(8)$ & $2841(5)$ & $6267(3)$ & $48(2)$ \\
\hline $0(4)$ & $145(8)$ & $3213(6)$ & $5189(4)$ & $54(2)$ \\
\hline $0(5)$ & $8768(9)$ & $2725(6)$ & $1889(3)$ & $55(2)$ \\
\hline $0(6)$ & $7902(8)$ & $3071(5)$ & $786(4)$ & $52(2)$ \\
\hline $0(7)$ & $3790(8)$ & $2366(6)$ & $-1265(4)$ & $60(2)$ \\
\hline $0(8)$ & $5233(9)$ & $3190(6)$ & $-270(4)$ & $59(2)$ \\
\hline$N(1)$ & $2568(10)$ & $1027(6)$ & $3971(4)$ & $40(2)$ \\
\hline$N(2)$ & $2747(10)$ & $-556(7)$ & $4886(4)$ & $43(2)$ \\
\hline$N(3)$ & $2104(10)$ & $139(6)$ & $5104(4)$ & $36(2)$ \\
\hline$N(4)$ & $886(9)$ & $1635(6)$ & $5552(4)$ & $39(2)$ \\
\hline$N(5)$ & $8684(9)$ & 1519 (6) & $917(4)$ & $40(2)$ \\
\hline$N(6)$ & $7847(9)$ & $-516(6)$ & $106(4)$ & $40(2)$ \\
\hline$N(7)$ & $7102(9)$ & $111(6)$ & $-125(3)$ & $36(2)$ \\
\hline$N(8)$ & $5557(9)$ & 1461(6) & $-578(4)$ & $37(2)$ \\
\hline$c(1)$ & $3300(10)$ & $201(7)$ & $3864(4)$ & $30(2)$ \\
\hline$C(2)$ & $4033(11)$ & $58(7)$ & $3344(5)$ & $38(2)$ \\
\hline$C(3)$ & $4732(12)$ & $-771(8)$ & $3247(5)$ & $47(3)$ \\
\hline$c(4)$ & $4695(12)$ & $-1511(8)$ & $3683(5)$ & $49(3)$ \\
\hline$C(5)$ & $4032(13)$ & $-1402(8)$ & $4191(5)$ & $47(3)$ \\
\hline$c(6)$ & $3287(11)$ & $-544(7)$ & $4310(4)$ & $36(2)$ \\
\hline$c(7)$ & $1671(10)$ & $49(7)$ & $5731(4)$ & $32(2)$ \\
\hline$C(8)$ & $1806(11)$ & $-811(7)$ & $6065(5)$ & $39(2)$ \\
\hline$c(9)$ & $1427(12)$ & $-825(9)$ & $6647(5)$ & $48(3)$ \\
\hline$C(10)$ & $880(14)$ & $23(9)$ & $6920(5)$ & $54(3)$ \\
\hline$C(11)$ & $739(12)$ & $874(8)$ & $6596(4)$ & $44(3)$ \\
\hline$c(12)$ & 1091(11) & $888(7)$ & $5973(4)$ & $32(2)$ \\
\hline$C(13)$ & $4546(11)$ & $2682(7)$ & $3673(4)$ & $35(2)$ \\
\hline$C(14)$ & $5169(13)$ & $2853(8)$ & $3124(5)$ & $45(3)$ \\
\hline$c(15)$ & $6680(13)$ & $3330(8)$ & $3161(5)$ & $50(3)$ \\
\hline$C(16)$ & $7583(11)$ & $3635(7)$ & $3727(5)$ & $44(3)$ \\
\hline$c(17)$ & $6914(12)$ & $3424(8)$ & $4269(5)$ & $46(3)$ \\
\hline$c(18)$ & $5434(12)$ & $2965(8)$ & $4253(5)$ & $42(3)$ \\
\hline$C(19)$ & $9205(12)$ & $4138(9)$ & $3754(6)$ & $60(3)$ \\
\hline$C(20)$ & $2514(10)$ & $3479(7)$ & $6022(4)$ & $32(2)$ \\
\hline$c(21)$ & $3419(12)$ & $3851(8)$ & $5592(5)$ & $43(3)$ \\
\hline$C(22)$ & $4890(12)$ & 4298(8) & $5797(5)$ & $45(3)$ \\
\hline$C(23)$ & $5509(11)$ & $4415(7)$ & $6414(5)$ & $38(2)$ \\
\hline$c(24)$ & $4592(12)$ & $4068(7)$ & $6863(5)$ & $40(3)$ \\
\hline$C(25)$ & $3078(11)$ & $3588(7)$ & $6666(5)$ & $38(2)$ \\
\hline$c(26)$ & $7196(12)$ & $4882(8)$ & $6630(5)$ & $50(3)$ \\
\hline
\end{tabular}




\begin{tabular}{lrrrr}
$C(27)$ & $9365(11)$ & $674(7)$ & $1054(4)$ & $35(2)$ \\
$C(28)$ & $10503(11)$ & $707(7)$ & $1575(4)$ & $33(2)$ \\
$C(29)$ & $11160(12)$ & $-129(8)$ & $1691(5)$ & $43(3)$ \\
$C(30)$ & $10711(13)$ & $-1053(9)$ & $1309(5)$ & $52(3)$ \\
$C(31)$ & $9635(13)$ & $-1118(8)$ & $819(5)$ & $48(3)$ \\
$C(32)$ & $8935(11)$ & $-257(8)$ & $668(4)$ & $38(2)$ \\
$C(33)$ & $6087(10)$ & $-253(7)$ & $-694(4)$ & $28(2)$ \\
$C(34)$ & $5883(11)$ & $-1247(8)$ & $-971(4)$ & $38(2)$ \\
$C(35)$ & $4885(13)$ & $-1504(9)$ & $-1529(5)$ & $49(3)$ \\
$C(36)$ & $4156(13)$ & $-749(8)$ & $-1786(5)$ & $45(3)$ \\
$C(37)$ & $4331(12)$ & $229(8)$ & $-1517(4)$ & $41(3)$ \\
$C(38)$ & $5340(11)$ & $504(7)$ & $-939(4)$ & $33(2)$ \\
$C(39)$ & $10852(12)$ & $3242(7)$ & $1188(5)$ & $38(2)$ \\
$C(40)$ & $11889(12)$ & $3510(7)$ & $1730(5)$ & $43(3)$ \\
$C(41)$ & $13353(13)$ & $3972(8)$ & $1680(5)$ & $46(3)$ \\
$C(42)$ & $13814(13)$ & $4165(7)$ & $1111(5)$ & $45(3)$ \\
$C(43)$ & $12786(12)$ & $3886(8)$ & $566(5)$ & $46(3)$ \\
$C(44)$ & $11244(13)$ & $3414(8)$ & $599(5)$ & $46(3)$ \\
$C(45)$ & $15437(12)$ & $4668(8)$ & $1059(6)$ & $54(3)$ \\
$C(46)$ & $6690(10)$ & $3027(7)$ & $-1214(4)$ & $31(2)$ \\
$C(47)$ & $7973(11)$ & $3629(8)$ & $-869(5)$ & $40(3)$ \\
$C(48)$ & $9238(13)$ & $3992(7)$ & $-1177(4)$ & $41(3)$ \\
$C(49)$ & $9184(12)$ & $3759(8)$ & $-1815(5)$ & $45(3)$ \\
$C(50)$ & $7873(13)$ & $3169(8)$ & $-2145(5)$ & $48(3)$ \\
$C(51)$ & $6632(13)$ & $2790(8)$ & $-1851(5)$ & $43(3)$ \\
$C(52)$ & $10576(15)$ & $4127(11)$ & $-2145(6)$ & $74(4)$ \\
\hline
\end{tabular}

Table 3. Bond lengths [A] and angles [deg] for ad96a.

\begin{tabular}{ll}
\hline$P d(1)-N(3)$ & $1.931(8)$ \\
$P d(1)-N(1)$ & $1.968(9)$ \\
$P d(1)-N(4)$ & $2.020(8)$ \\
$P d(1)-O(1)$ & $2.279(7)$ \\
$P d(1)-S(1)$ & $2.797(3)$ \\
$P d(2)-N(7)$ & $1.957(8)$ \\
$P d(2)-N(5)$ & $1.970(8)$ \\
$P d(2)-N(8)$ & $2.030(8)$ \\
$P d(2)-O(6)$ & $2.300(7)$ \\
$P d(2)-S(3)$ & $2.811(3)$ \\
$S(1)-O(2)$ & $1.427(7)$ \\
$S(1)-O(1)$ & $1.469(7)$ \\
$S(1)-N(1)$ & $1.616(8)$ \\
$S(1)-C(13)$ & $1.758(10)$ \\
$S(2)-O(4)$ & $1.426(8)$ \\
$S(2)-O(3)$ & $1.439(7)$ \\
$S(2)-N(4)$ & $1.643(8)$ \\
$S(2)-C(20)$ & $1.777(9)$ \\
$S(3)-O(5)$ & $1.430(7)$ \\
$S(3)-O(6)$ & $1.487(7)$ \\
$S(3)-N(5)$ & $1.584(9)$ \\
$S(3)-C(39)$ & $1.770(11)$ \\
$S(4)-O(8)$ & $1.420(8)$ \\
$S(4)-O(7)$ & $1.449(8)$ \\
$S(4)-N(8)$ & $1.619(8)$ \\
&
\end{tabular}




\begin{tabular}{|c|c|}
\hline$S(4)-C(46)$ & $1.764(9)$ \\
\hline$N(1)-C(1)$ & $1.393(12)$ \\
\hline$N(2)-N(3)$ & $1.264(11)$ \\
\hline$N(2)-C(6)$ & $1.387(13)$ \\
\hline$N(3)-C(7)$ & $1.459(12)$ \\
\hline$N(4)-C(12)$ & $1.408(12)$ \\
\hline$N(5)-C(27)$ & $1.396(12)$ \\
\hline$N(6)-N(7)$ & $1.245(11)$ \\
\hline$N(6)-C(32)$ & $1.445(12)$ \\
\hline$N(7)-C(33)$ & 1. $441(11)$ \\
\hline$N(8)-C(38)$ & $1.419(12)$ \\
\hline$C(1)-C(2)$ & $1.387(13)$ \\
\hline$C(1)-C(6)$ & $1.427(13)$ \\
\hline$c(2)-c(3)$ & $1.374(14)$ \\
\hline$C(3)-C(4)$ & $1.404(15)$ \\
\hline$C(4)-C(5)$ & $1.322(15)$ \\
\hline$c(5)-c(6)$ & $1.440(15)$ \\
\hline$C(7)-C(8)$ & $1.405(13)$ \\
\hline$C(7)-C(12)$ & $1.405(13)$ \\
\hline$C(8)-C(9)$ & $1.341(14)$ \\
\hline$C(9)-C(10)$ & $1.424(16)$ \\
\hline$C(10)-C(11)$ & $1.384(15)$ \\
\hline$C(11)-C(12)$ & $1.418(13)$ \\
\hline$C(13)-C(14)$ & $1.389(14)$ \\
\hline$C(13)-C(18)$ & $1.392(13)$ \\
\hline$C(14)-C(15)$ & $1.394(15)$ \\
\hline$C(15)-C(16)$ & $1.377(15)$ \\
\hline$C(16)-C(17)$ & $1.404(15)$ \\
\hline$C(16)-C(19)$ & $1.497(14)$ \\
\hline$C(17)-C(18)$ & $1.368(14)$ \\
\hline$C(20)-C(21)$ & $1.375(14)$ \\
\hline$C(20)-C(25)$ & $1.407(13)$ \\
\hline$C(21)-C(22)$ & $1.366(14)$ \\
\hline$C(22)-C(23)$ & $1.367(14)$ \\
\hline$C(23)-C(24)$ & $1.402(14)$ \\
\hline$C(23)-C(26)$ & $1.547(14)$ \\
\hline$C(24)-C(25)$ & $1.408(14)$ \\
\hline$C(27)-C(32)$ & $1.414(13)$ \\
\hline$C(27)-C(28)$ & $1.418(13)$ \\
\hline$C(28)-C(29)$ & $1.366(14)$ \\
\hline$C(29)-C(30)$ & $1.405(15)$ \\
\hline$C(30)-C(31)$ & $1.330(15)$ \\
\hline$C(31)-C(32)$ & $1.433(14)$ \\
\hline$C(33)-C(34)$ & $1.387(13)$ \\
\hline$C(33)-C(38)$ & $1.397(13)$ \\
\hline$C(34)-C(35)$ & $1.398(14)$ \\
\hline$C(35)-C(36)$ & $1.395(15)$ \\
\hline$C(36)-C(37)$ & $1.365(14)$ \\
\hline$C(37)-C(38)$ & $1.437(13)$ \\
\hline$C(39)-C(44)$ & $1.381(14)$ \\
\hline$C(39)-C(40)$ & $1.391(14)$ \\
\hline$C(40)-C(41)$ & $1.381(15)$ \\
\hline$C(41)-C(42)$ & $1.371(15)$ \\
\hline$C(42)-C(43)$ & $1.391(15)$ \\
\hline$C(42)-C(45)$ & $1.526(15)$ \\
\hline$C(43)-C(44)$ & $1.441(15)$ \\
\hline$C(46)-C(51)$ & $1.379(13)$ \\
\hline
\end{tabular}


$C(46)-C(47)$
$C(47)-C(48)$
$C(48)-C(49)$
$C(49)-C(50)$
$C(49)-C(52)$
$C(50)-C(51)$

$\mathrm{N}(3)-\mathrm{Pd}(1)-\mathrm{N}(1)$

$\mathrm{N}(3)-\mathrm{Pd}(1)-\mathrm{N}(4)$

$\mathrm{N}(1)-\mathrm{Pd}(1)-\mathrm{N}(4)$

$\mathrm{N}(3)-\mathrm{Pd}(1)-0(1)$

$\mathrm{N}(1)-\mathrm{Pd}(1)-0(1)$

$\mathrm{N}(4)-\mathrm{Pd}(1)-0(1)$

$\mathrm{N}(3)-\mathrm{Pd}(1)-\mathrm{S}(1)$

$\mathrm{N}(1)-\mathrm{Pd}(1)-\mathrm{S}(1)$

$\mathrm{N}(4)-\mathrm{Pd}(1)-\mathrm{S}(1)$

0 (1) $-\mathrm{Pd}(1)-\mathrm{S}(1)$

$\mathrm{N}(7)-\mathrm{Pd}(2)-\mathrm{N}(5)$

$\mathrm{N}(7)-\mathrm{Pd}(2)-\mathrm{N}(8)$

$\mathrm{N}(5)-\mathrm{Pd}(2)-\mathrm{N}(8)$

$\mathrm{N}(7)-\mathrm{Pd}(2)-0(6)$

$\mathrm{N}(5)-\mathrm{Pd}(2)-0(6)$

$\mathrm{N}(8)-\mathrm{Pd}(2)-\mathrm{O}(6)$

$\mathrm{N}(7)-\mathrm{Pd}(2)-\mathrm{S}(3)$

$\mathrm{N}(5)-\mathrm{Pd}(2)-\mathrm{S}(3)$

$\mathrm{N}(8)-\mathrm{Pd}(2)-\mathrm{S}(3)$

0 (6) $-\mathrm{Pd}(2)-\mathrm{S}(3)$

$O(2)-S(1)-0(1)$

$\mathrm{O}(2)-\mathrm{S}(1)-\mathrm{N}(1)$

$O(1)-S(1)-N(1)$

$0(2)-S(1)-C(13)$

$0(1)-S(1)-C(13)$

$\mathrm{N}(1)-\mathrm{S}(1)-\mathrm{C}(13)$

$0(2)-S(1)-P d(1)$

0 (1) - S (1) - Pd(1)

$\mathrm{N}(1)-\mathrm{S}(1)-\mathrm{Pd}(1)$

$\mathrm{C}(13)-\mathrm{S}(1)-\mathrm{Pd}(1)$

$0(4)-S(2)-O(3)$

$\mathrm{O}(4)-\mathrm{S}(2)-\mathrm{N}(4)$

$\mathrm{O}(3)-\mathrm{S}(2)-\mathrm{N}(4)$

$\mathrm{O}(4)-\mathrm{S}(2)-\mathrm{C}(20)$

$\mathrm{O}(3)-\mathrm{S}(2)-\mathrm{C}(20)$

$\mathrm{N}(4)-\mathrm{S}(2)-\mathrm{C}(20)$

$0(5)-S(3)-0(6)$

$\mathrm{O}(5)-\mathrm{S}(3)-\mathrm{N}(5)$

$O(6)-S(3)-N(5)$

$0(5)-S(3)-C(39)$

$\mathrm{O}(6)-\mathrm{S}(3)-\mathrm{C}(39)$

$\mathrm{N}(5)-\mathrm{S}(3)-\mathrm{C}(39)$

$O(5)-S(3)-P d(2)$

$\mathrm{O}(6)-\mathrm{S}(3)-\mathrm{Pd}(2)$

$\mathrm{N}(5)-\mathrm{S}(3)-\mathrm{Pd}(2)$

$\mathrm{C}(39)-\mathrm{S}(3)-\mathrm{Pd}(2)$

$0(8)-S(4)-0(7)$

$\mathrm{O}(8)-\mathrm{S}(4)-\mathrm{N}(8)$

$0(7)-S(4)-N(8)$

$\mathrm{O}(8)-\mathrm{S}(4)-\mathrm{C}(46)$
1. 392(13)

$1.422(14)$

$1.383(14)$

$1.389(15)$

$1.545(15)$

1.394(15)

93.1(3)

$82.7(3)$

$175.3(3)$

$158.5(3)$

$65.8(3)$

$118.5(3)$

$127.0(2)$

$34.4(2)$

$150.0(2)$

$31.60(18)$

$94.8(3)$

$81.9(3)$

$176.4(3)$

$159.7(3)$

$65.0(3)$

118.3(3)

127.9(2)

$33.1(3)$

150.2(2)

$31.87(18)$

$117.2(4)$

$114.5(4)$

97.5(4)

$107.9(4)$

$109.6(4)$

$109.7(4)$

$135.7(3)$

$54.4(3)$

$43.5(3)$

$115.8(3)$

$118.4(5)$

105.9(4)

$112.3(4)$

$106.4(4)$

$108.4(4)$

$104.5(4)$

$118.3(5)$

$114.6(4)$

$97.6(4)$

$107.5(5)$

$109.2(5)$

109.1(5)

131.8(3)

$54.8(3)$

42.8(3)

$119.9(3)$

$117.6(5)$

105.7(5)

$111.9(5)$

107.3(5) 


\begin{tabular}{|c|c|}
\hline $0(7)-S(4)-C(46)$ & $107.8(5)$ \\
\hline$N(8)-S(4)-C(46)$ & $105.9(4)$ \\
\hline $\mathrm{S}(1)-0(1)-\mathrm{Pd}(1)$ & $94.0(3)$ \\
\hline$S(3)-0(6)-P d(2)$ & $93.4(3)$ \\
\hline$C(1)-N(1)-S(1)$ & $129.3(7)$ \\
\hline$C(1)-N(1)-P d(1)$ & $125.9(6)$ \\
\hline$S(1)-N(1)-P d(1)$ & $102.1(4)$ \\
\hline$N(3)-N(2)-C(6)$ & $123.4(9)$ \\
\hline$N(2)-N(3)-C(7)$ & $117.1(8)$ \\
\hline$N(2)-N(3)-P d(1)$ & $128.4(7)$ \\
\hline $\mathrm{C}(7)-\mathrm{N}(3)-\mathrm{Pd}(1)$ & $114.3(6)$ \\
\hline$C(12)-N(4)-S(2)$ & $124.6(7)$ \\
\hline$C(12)-N(4)-P d(1)$ & $112.9(6)$ \\
\hline$S(2)-N(4)-P d(1)$ & $118.0(4)$ \\
\hline$C(27)-N(5)-S(3)$ & $131.7(7)$ \\
\hline$C(27)-N(5)-P d(2)$ & $124.1(6)$ \\
\hline$S(3)-N(5)-P d(2)$ & $104.0(4)$ \\
\hline$N(7)-N(6)-C(32)$ & $122.4(8)$ \\
\hline$N(6)-N(7)-C(33)$ & $116.0(8)$ \\
\hline$N(6)-N(7)-P d(2)$ & $128.2(7)$ \\
\hline$C(33)-N(7)-P d(2)$ & $115.8(6)$ \\
\hline$C(38)-N(8)-S(4)$ & $126.8(7)$ \\
\hline$C(38)-N(8)-P d(2)$ & $111.6(6)$ \\
\hline$S(4)-N(8)-P d(2)$ & $117.8(5)$ \\
\hline$C(2)-C(1)-N(1)$ & $123.6(8)$ \\
\hline$C(2)-C(1)-C(6)$ & $118.2(9)$ \\
\hline$N(1)-C(1)-C(6)$ & $118.2(8)$ \\
\hline$C(3)-C(2)-C(1)$ & $122.3(10)$ \\
\hline$C(2)-C(3)-C(4)$ & $119.3(10)$ \\
\hline$C(5)-C(4)-C(3)$ & $120.8(10)$ \\
\hline$C(4)-C(5)-C(6)$ & $121.6(10)$ \\
\hline$N(2)-C(6)-C(1)$ & $130.3(9)$ \\
\hline$N(2)-C(6)-C(5)$ & $111.8(9)$ \\
\hline$C(1)-C(6)-C(5)$ & $117.8(9)$ \\
\hline$C(8)-C(7)-C(12)$ & $122.5(9)$ \\
\hline$C(8)-C(7)-N(3)$ & $122.9(9)$ \\
\hline $\mathrm{C}(12)-\mathrm{C}(7)-\mathrm{N}(3)$ & $114.6(8)$ \\
\hline$C(9)-C(8)-C(7)$ & $119.5(10)$ \\
\hline$C(8)-C(9)-C(10)$ & $120.1(10)$ \\
\hline$C(11)-C(10)-C(9)$ & $121.1(10)$ \\
\hline$C(10)-C(11)-C(12)$ & $119.6(10)$ \\
\hline$C(7)-C(12)-N(4)$ & $115.0(8)$ \\
\hline$C(7)-C(12)-C(11)$ & $117.2(9)$ \\
\hline$N(4)-C(12)-C(11)$ & $127.7(9)$ \\
\hline$C(14)-C(13)-C(18)$ & $120.1(10)$ \\
\hline$C(14)-C(13)-S(1)$ & $119.5(7)$ \\
\hline$C(18)-C(13)-S(1)$ & $120.4(8)$ \\
\hline$C(13)-C(14)-C(15)$ & $119.3(10)$ \\
\hline$C(16)-C(15)-C(14)$ & $122.1(10)$ \\
\hline$C(15)-C(16)-C(17)$ & $116.6(9)$ \\
\hline$C(15)-C(16)-C(19)$ & $121.0(11)$ \\
\hline$C(17)-C(16)-C(19)$ & $122.4(10)$ \\
\hline$C(18)-C(17)-C(16)$ & $123.1(9)$ \\
\hline$C(17)-C(18)-C(13)$ & $118.8(10)$ \\
\hline$C(21)-C(20)-C(25)$ & $120.5(9)$ \\
\hline$C(21)-C(20)-S(2)$ & $119.5(7)$ \\
\hline$C(25)-C(20)-S(2)$ & $120.0(7)$ \\
\hline
\end{tabular}




$\begin{array}{ll}C(22)-C(21)-C(20) & 118.9(10) \\ C(21)-C(22)-C(23) & 123.3(10) \\ C(22)-C(23)-C(24) & 118.7(9) \\ C(22)-C(23)-C(26) & 122.2(9) \\ C(24)-C(23)-C(26) & 119.1(9) \\ C(23)-C(24)-C(25) & 119.3(9) \\ C(20)-C(25)-C(24) & 119.2(9) \\ N(5)-C(27)-C(32) & 120.5(8) \\ N(5)-C(27)-C(28) & 122.3(9) \\ C(32)-C(27)-C(28) & 117.3(9) \\ C(29)-C(28)-C(27) & 120.8(9) \\ C(28)-C(29)-C(30) & 121.4(10) \\ C(31)-C(30)-C(29) & 119.7(11) \\ C(30)-C(31)-C(32) & 121.1(11) \\ C(27)-C(32)-C(31) & 119.7(9) \\ C(27)-C(32)-N(6) & 130.1(9) \\ C(31)-C(32)-N(6) & 110.2(9) \\ C(34)-C(33)-C(38) & 123.8(8) \\ C(34)-C(33)-N(7) & 123.6(8) \\ C(38)-C(33)-N(7) & 112.7(8) \\ C(33)-C(34)-C(35) & 118.2(9) \\ C(36)-C(35)-C(34) & 118.6(10) \\ C(37)-C(36)-C(35) & 124.0(9) \\ C(36)-C(37)-C(38) & 118.1(9) \\ C(33)-C(38)-N(8) & 117.6(8) \\ C(33)-C(38)-C(37) & 117.3(8) \\ N(8)-C(38)-C(37) & 125.0(9) \\ C(44)-C(39)-C(40) & 122.0(10) \\ C(44)-C(39)-S(3) & 118.2(8) \\ C(40)-C(39)-S(3) & 119.8(8) \\ C(41)-C(40)-C(39) & 119.0(10) \\ C(42)-C(41)-C(40) & 121.8(10) \\ C(41)-C(42)-C(43) & 119.4(10) \\ C(41)-C(42)-C(45) & 121.5(10) \\ C(43)-C(42)-C(45) & 119.1(10) \\ C(42)-C(43)-C(44) & 120.4(10) \\ C(39)-C(44)-C(43) & 117.3(10) \\ C(51)-C(46)-C(47) & 120.6(9) \\ C(51)-C(46)-S(4) & 120.4(7) \\ C(47)-C(46)-S(4) & 118.9(7) \\ C(46)-C(47)-C(48) & 119.4(9) \\ C(49)-C(48)-C(47) & 120.2(10) \\ C(48)-C(49)-C(50) & 118.7(10) \\ C(48)-C(49)-C(52) & 120.1(10) \\ C(50)-C(49)-C(52) & 121.2(10) \\ C(49)-C(50)-C(51) & 122.0(10) \\ C(46)-C(51)-C(50) & 119.1(10) \\ & \end{array}$

Symmetry transformations used to generate equivalent atoms:

Table 4. Anisotropic displacement parameters $\left(A^{\wedge} 2 \times 10 \wedge 3\right)$ for ad96a. The anisotropic displacement factor exponent takes the form:

-2 pi^2 [ h^2 a*^2 U11 + .. + $2 \mathrm{~h} k a^{*} b^{*} \mathrm{U} 12$ ] 


\begin{tabular}{|c|c|c|c|c|c|c|}
\hline & U11 & U22 & U33 & U23 & U13 & U12 \\
\hline $\operatorname{Pd}(1)$ & $27(1)$ & $26(1)$ & $32(1)$ & $3(1)$ & $5(1)$ & $5(1)$ \\
\hline $\mathrm{Pd}(2)$ & $30(1)$ & $28(1)$ & $32(1)$ & $4(1)$ & $3(1)$ & $5(1)$ \\
\hline$S(1)$ & $33(1)$ & $37(1)$ & $38(1)$ & $10(1)$ & $4(1)$ & $7(1)$ \\
\hline$s(2)$ & $28(1)$ & $35(1)$ & $50(2)$ & $-1(1)$ & $3(1)$ & $5(1)$ \\
\hline$S(3)$ & $41(2)$ & $39(1)$ & $48(2)$ & $4(1)$ & $8(1)$ & $9(1)$ \\
\hline$s(4)$ & $37(1)$ & $42(1)$ & $52(2)$ & $7(1)$ & $11(1)$ & $13(1)$ \\
\hline $0(1)$ & $26(4)$ & $41(4)$ & $52(4)$ & $1(3)$ & $3(3)$ & $5(3)$ \\
\hline $0(2)$ & $42(4)$ & $52(4)$ & $45(4)$ & $19(3)$ & $-1(3)$ & $-4(4)$ \\
\hline $0(3)$ & $29(4)$ & $55(4)$ & $60(5)$ & $-9(4)$ & $18(3)$ & $7(3)$ \\
\hline $0(4)$ & $38(4)$ & $53(4)$ & $67(5)$ & $-1(4)$ & $-7(4)$ & $8(4)$ \\
\hline $0(5)$ & $65(5)$ & $52(4)$ & $48(4)$ & $-7(4)$ & $26(4)$ & $1(4)$ \\
\hline $0(6)$ & $41(4)$ & $51(4)$ & $68(5)$ & $12(4)$ & $2(4)$ & $21(4)$ \\
\hline $0(7)$ & $23(4)$ & $66(5)$ & $91(6)$ & $16(5)$ & $-4(4)$ & $12(4)$ \\
\hline $0(8)$ & $60(5)$ & $59(5)$ & $62(5)$ & $-7(4)$ & $25(4)$ & $10(4)$ \\
\hline$N(1)$ & $46(5)$ & $28(4)$ & $47(5)$ & $8(4)$ & $3(4)$ & $7(4)$ \\
\hline$N(2)$ & $41(5)$ & $48(5)$ & $38(5)$ & $3(4)$ & $2(4)$ & $1(4)$ \\
\hline$N(3)$ & $48(5)$ & $28(4)$ & $31(4)$ & $-7(3)$ & $-2(4)$ & $11(4)$ \\
\hline$N(4)$ & $31(5)$ & $37(4)$ & $44(5)$ & $-2(4)$ & $1(4)$ & $-2(4)$ \\
\hline$N(5)$ & $33(5)$ & $48(5)$ & $39(5)$ & $8(4)$ & $-3(4)$ & $7(4)$ \\
\hline$N(6)$ & $32(5)$ & $42(5)$ & $51(5)$ & $13(4)$ & $12(4)$ & $11(4)$ \\
\hline$N(7)$ & $38(5)$ & $43(5)$ & $34(4)$ & $15(4)$ & $22(4)$ & $9(4)$ \\
\hline$N(8)$ & $26(4)$ & $43(5)$ & $51(5)$ & $12(4)$ & $19(4)$ & $14(4)$ \\
\hline$c(1)$ & $28(5)$ & $31(5)$ & $29(5)$ & $3(4)$ & $-1(4)$ & $2(4)$ \\
\hline$c(2)$ & $38(6)$ & $37(5)$ & $40(5)$ & $-1(4)$ & $14(5)$ & $6(4)$ \\
\hline$c(3)$ & $37(6)$ & $44(6)$ & $55(7)$ & $-15(5)$ & $4(5)$ & $1(5)$ \\
\hline$c(4)$ & $33(6)$ & $48(6)$ & $67(7)$ & $-10(6)$ & $6(5)$ & $15(5)$ \\
\hline$c(5)$ & $48(7)$ & $36(6)$ & $52(6)$ & $5(5)$ & $-8(5)$ & $\odot(5)$ \\
\hline$C(6)$ & $35(6)$ & $33(5)$ & $37(5)$ & $-6(4)$ & $4(4)$ & $-2(4)$ \\
\hline$c(7)$ & $18(5)$ & $41(5)$ & $33(5)$ & $11(4)$ & $-3(4)$ & $-6(4)$ \\
\hline$C(8)$ & $24(5)$ & $37(5)$ & $52(6)$ & $8(5)$ & $5(5)$ & $-1(4)$ \\
\hline$C(9)$ & $43(6)$ & $55(6)$ & $44(6)$ & $24(5)$ & $-1(5)$ & $-3(5)$ \\
\hline$c(10)$ & $56(7)$ & $61(7)$ & $50(7)$ & $14(6)$ & $17(6)$ & $12(6)$ \\
\hline$C(11)$ & $40(6)$ & $57(6)$ & $31(5)$ & $\odot(5)$ & $6(5)$ & $-1(5)$ \\
\hline$C(12)$ & $35(5)$ & $32(5)$ & $28(5)$ & $5(4)$ & $9(4)$ & $\odot(4)$ \\
\hline$c(13)$ & $4 \odot(6)$ & $29(5)$ & $32(5)$ & $3(4)$ & $-5(4)$ & $4(4)$ \\
\hline$C(14)$ & $49(7)$ & $46(6)$ & $39(6)$ & $7(5)$ & $7(5)$ & $4(5)$ \\
\hline$C(15)$ & $49(7)$ & $54(6)$ & $56(7)$ & $21(5)$ & $19(6)$ & $13(5)$ \\
\hline$C(16)$ & $26(5)$ & $38(5)$ & $74(7)$ & $25(5)$ & $5(5)$ & $12(4)$ \\
\hline$C(17)$ & $29(6)$ & $54(6)$ & $47(6)$ & $9(5)$ & $-12(5)$ & $\odot(5)$ \\
\hline$C(18)$ & $35(6)$ & $47(6)$ & $43(6)$ & $15(5)$ & $4(5)$ & $1(5)$ \\
\hline$C(19)$ & $25(6)$ & $57(7)$ & $97(9)$ & $17(7)$ & $\odot(6)$ & $4(5)$ \\
\hline$C(20)$ & $24(5)$ & $31(5)$ & $44(5)$ & $10(4)$ & $4(4)$ & $8(4)$ \\
\hline$c(21)$ & $44(6)$ & $42(6)$ & $37(5)$ & $3(5)$ & $2(5)$ & $-4(5)$ \\
\hline$C(22)$ & $28(5)$ & $41(6)$ & $64(7)$ & $7(5)$ & $7(5)$ & $\odot(5)$ \\
\hline$C(23)$ & $30(5)$ & $30(5)$ & $52(6)$ & $3(5)$ & $1(5)$ & $-1(4)$ \\
\hline$c(24)$ & $39(6)$ & $30(5)$ & $49(6)$ & $-8(5)$ & $-5(5)$ & $11(4)$ \\
\hline$C(25)$ & $38(6)$ & $32(5)$ & $47(6)$ & $-6(4)$ & $19(5)$ & $11(4)$ \\
\hline$C(26)$ & $39(6)$ & $39(6)$ & $67(7)$ & $2(5)$ & $\odot(6)$ & 1(5) \\
\hline$c(27)$ & $41(6)$ & $35(5)$ & $30(5)$ & $12(4)$ & $11(4)$ & $1(4)$ \\
\hline$C(28)$ & $35(5)$ & $38(5)$ & $21(4)$ & $0(4)$ & $5(4)$ & $-4(4)$ \\
\hline$C(29)$ & $35(6)$ & $56(6)$ & $41(6)$ & $14(5)$ & $8(5)$ & $7(5)$ \\
\hline$c(30)$ & $42(7)$ & $54(7)$ & $58(7)$ & $3(6)$ & $5(6)$ & $9(5)$ \\
\hline
\end{tabular}




\begin{tabular}{llllrrr}
$C(31)$ & $56(7)$ & $50(6)$ & $43(6)$ & $9(5)$ & $15(5)$ & $16(6)$ \\
$C(32)$ & $32(5)$ & $53(6)$ & $29(5)$ & $11(4)$ & $2(4)$ & $10(5)$ \\
$C(33)$ & $18(4)$ & $31(5)$ & $32(5)$ & $13(4)$ & $1(4)$ & $-4(4)$ \\
$C(34)$ & $32(5)$ & $47(6)$ & $44(5)$ & $21(5)$ & $18(4)$ & $14(4)$ \\
$C(35)$ & $48(7)$ & $51(6)$ & $39(6)$ & $-6(5)$ & $2(5)$ & $-12(5)$ \\
$C(36)$ & $47(7)$ & $48(6)$ & $31(5)$ & $8(5)$ & $-7(5)$ & $-9(5)$ \\
$C(37)$ & $41(6)$ & $50(6)$ & $35(5)$ & $18(5)$ & $8(5)$ & $8(5)$ \\
$C(38)$ & $37(5)$ & $35(5)$ & $26(5)$ & $6(4)$ & $8(4)$ & $-4(4)$ \\
$C(39)$ & $43(6)$ & $31(5)$ & $40(5)$ & $2(4)$ & $4(5)$ & $9(4)$ \\
$C(40)$ & $49(7)$ & $31(5)$ & $46(6)$ & $6(5)$ & $4(5)$ & $-1(5)$ \\
$C(41)$ & $49(7)$ & $43(6)$ & $43(6)$ & $3(5)$ & $0(5)$ & $6(5)$ \\
$C(42)$ & $50(7)$ & $26(5)$ & $57(7)$ & $-6(5)$ & $10(6)$ & $2(5)$ \\
$C(43)$ & $39(6)$ & $42(6)$ & $60(7)$ & $1(5)$ & $16(5)$ & $11(5)$ \\
$C(44)$ & $55(7)$ & $41(6)$ & $46(6)$ & $4(5)$ & $16(5)$ & $17(5)$ \\
$C(45)$ & $36(6)$ & $43(6)$ & $78(8)$ & $-4(6)$ & $13(6)$ & $-4(5)$ \\
$C(46)$ & $29(5)$ & $25(4)$ & $39(5)$ & $3(4)$ & $4(4)$ & $1(4)$ \\
$C(47)$ & $30(5)$ & $50(6)$ & $41(6)$ & $4(5)$ & $7(5)$ & $5(5)$ \\
$C(48)$ & $51(7)$ & $39(5)$ & $35(5)$ & $-1(4)$ & $6(5)$ & $13(5)$ \\
$C(49)$ & $39(6)$ & $48(6)$ & $47(6)$ & $2(5)$ & $12(5)$ & $0(5)$ \\
$C(50)$ & $64(8)$ & $41(6)$ & $43(6)$ & $14(5)$ & $12(5)$ & $12(5)$ \\
$C(51)$ & $48(6)$ & $41(6)$ & $39(6)$ & $13(5)$ & $-2(5)$ & $10(5)$ \\
$C(52)$ & $59(8)$ & $80(9)$ & $84(9)$ & $0(8)$ & $39(7)$ & $-3(7)$ \\
& & & & & & \\
\hline
\end{tabular}

Table 5. Hydrogen coordinates $(x 10 \wedge 4)$ and isotropic displacement parameters $\left(A^{\wedge} 2 \times 10 \wedge 3\right)$ for ad96a.

\begin{tabular}{lrrrl}
\hline & & & & \\
& $X$ & & & $U(\mathrm{eq})$ \\
\hline$H(2 C)$ & 4051 & 551 & 3044 & 45 \\
$H(3 B)$ & 5236 & -844 & 2889 & 56 \\
$H(4 B)$ & 5152 & -2095 & 3612 & 59 \\
$H(5 B)$ & 4046 & -1903 & 4485 & 57 \\
$H(8 B)$ & 2164 & -1378 & 5880 & 46 \\
$H(9 A)$ & 1523 & -1401 & 6877 & 58 \\
$H(10 A)$ & 607 & 4 & 7331 & 65 \\
$H(11 A)$ & 408 & 1445 & 6790 & 53 \\
$H(14 A)$ & 4573 & 2648 & 2727 & 54 \\
$H(15 A)$ & 7099 & 3448 & 2784 & 61 \\
$H(17 A)$ & 7518 & 3609 & 4667 & 55 \\
$H(18 A)$ & 5020 & 2842 & 4631 & 51 \\
$H(19 A)$ & 9811 & 3628 & 3632 & 90 \\
$H(19 B)$ & 9596 & 4439 & 4182 & 90 \\
$H(19 C)$ & 9271 & 4677 & 3465 & 90 \\
$H(21 A)$ & 3029 & 3799 & 5158 & 51 \\
$H(22 A)$ & 5514 & 4540 & 5495 & 54 \\
$H(24 A)$ & 4987 & 4156 & 7297 & 48 \\
$H(25 A)$ & 2446 & 3340 & 6964 & 45 \\
$H(26 A)$ & 7843 & 4435 & 6463 & 74 \\
$H(26 B)$ & 7393 & 4949 & 7090 & 74 \\
$H(26 C)$ & 7423 & 5558 & 6475 & 74 \\
$H(28 A)$ & 10812 & 1318 & 1847 & 39 \\
$H(29 A)$ & 11936 & -84 & 2038 & 52 \\
& & & &
\end{tabular}




\begin{tabular}{lrrrr}
$H(30 A)$ & 11173 & -1628 & $140 \odot$ & 62 \\
$H(31 A)$ & 9325 & -1747 & 565 & 58 \\
$H(34 A)$ & 6408 & -1739 & -785 & 46 \\
$H(35 A)$ & 4706 & -2179 & -1730 & 58 \\
$H(36 A)$ & 3499 & -927 & -2171 & 54 \\
$H(37 A)$ & $380 \odot$ & 715 & -1707 & 49 \\
$H(40 A)$ & 11596 & 3377 & 2129 & 52 \\
$H(41 A)$ & 14061 & 4161 & 2051 & 55 \\
$H(43 A)$ & 13103 & 4007 & 169 & 55 \\
$H(44 A)$ & 10525 & 3229 & 232 & 55 \\
$H(45 A)$ & 15796 & 5199 & 1407 & 81 \\
$H(45 B)$ & 15459 & 4978 & 659 & 81 \\
$H(45 C)$ & 16097 & 4151 & 1077 & 81 \\
$H(47 A)$ & 8005 & 3795 & -431 & 48 \\
$H(48 A)$ & 10123 & 4396 & -943 & 50 \\
$H(50 A)$ & 7822 & 3020 & -2585 & 57 \\
$H(51 A)$ & 5758 & 2374 & -2085 & 51 \\
$H(52 A)$ & 10906 & 4866 & -2043 & 111 \\
$H(52 B)$ & 10301 & 3981 & $-260 \odot$ & 111 \\
$H(52 C)$ & 11405 & 3769 & $-200 \odot$ & 111 \\
\hline
\end{tabular}


For benzoquinoline complex $9 \mathbf{b}$.

\section{Structure Determination.}

Yellow needles of mr17 were grown from a chlorobenzene/diethyl ether solution at 22 deg. C. A crystal of dimensions $0.44 \times 0.06 \times 0.04 \mathrm{~mm}$ was mounted on a standard Bruker SMART 1K CCD-based X-ray diffractometer equipped with a LT-2 low temperature device and normal focus Mo-target X-ray tube $(\lambda=0.71073 \mathrm{~A})$ operated at $2000 \mathrm{~W}$ power $(50 \mathrm{kV}, 40 \mathrm{~mA})$. The X-ray intensities were measured at 123(2) K; the detector was placed at a distance $4.969 \mathrm{~cm}$ from the crystal. A total of 2740 frames were collected with a scan width of $0.5^{\circ}$ in $\omega$ and phi with an exposure time of $45 \mathrm{~s} /$ frame. The integration of the data yielded a total of 36204 reflections to a maximum $2 \theta$ value of $49.70^{\circ}$ of which 3897 were independent and 2847 were greater than $2 \sigma(\mathrm{I})$. The final cell constants (Table 1) were based on the xyz centroids of 6997 reflections above $10 \sigma(\mathrm{I})$. Analysis of the data showed negligible decay during data collection; the data were processed with SADABS and corrected fro absorption. The structure was solved and refined with the Bruker SHELXTL (version 6.12) software package, using the space group $\mathrm{C} 2 / \mathrm{c}$ with $\mathrm{Z}=8$ for the formula $\mathrm{C}_{25} \mathrm{H}_{20} \mathrm{~N}_{3} \mathrm{O}_{2} \mathrm{SClPd}$. All non-hydrogen atoms were refined anisotropically with the hydrogen atoms placed in idealized positions. Full matrix least-squares refinement based on $\mathrm{F}^{2}$ converged at $\mathrm{R} 1=0.0375$ and $\mathrm{wR} 2=0.0822$ [based on $\mathrm{I}>2 \operatorname{sigma}(\mathrm{I})$ ], $\mathrm{R} 1=0.0676$ and $\mathrm{wR} 2=0.0942$ for all data.

Sheldrick, G.M. SHELXTL, v. 6.12; Bruker Analytical X-ray, Madison, WI, 2001.

Sheldrick, G.M. SADABS, v. 2.10. Program for Empirical Absorption Correction of Area Detector Data, University of Gottingen: Gottingen, Germany, 2003.

Saint Plus, v. 7.01, Bruker Analytical X-ray, Madison, WI, 2003.

Table 1. Crystal data and structure refinement for mr17.

$\begin{array}{ll}\text { Identification code } & \mathrm{mr} 17 \\ \text { Empirical formula } & \mathrm{C} 25 \mathrm{H} 20 \mathrm{Cl} \mathrm{N} \text { 02 Pd S } \\ \text { Formula weight } & 568.35 \\ \text { Temperature } & 123(2) \mathrm{K} \\ \text { Wavelength } & 0.71073 \mathrm{~A} \\ \text { Crystal system, space group } & \text { Monoclinic, C2/C }\end{array}$


Unit cell dimensions

deg.

Volume

Z, Calculated density

Absorption coefficient

$F(\odot \odot \odot)$

Crystal size

Theta range for data collection

Limiting indices

Reflections collected / unique

Completeness to theta $=24.85$

Absorption correction

Max. and min. transmission

Refinement method

Data / restraints / parameters

Goodness-of-fit on $\mathrm{F}^{\wedge} 2$

Final $R$ indices [I $>2$ sigma(I)]

$\mathrm{R}$ indices (all data)

Largest diff. peak and hole $\mathrm{a}=31.742(2) \mathrm{A}$ alpha $=90 \mathrm{deg}$.

$\mathrm{b}=9.1460(6) \mathrm{A}$ beta $=127.054(1)$

$c=19.6641(12)$ A gamma $=90 \mathrm{deg}$.

$4555.9(5) \quad A^{\wedge} 3$

8, $1.657 \mathrm{Mg} / \mathrm{m}^{\wedge} 3$

$1.052 \mathrm{~mm}^{\wedge}-1$

2288

$0.44 \times 0.06 \times 0.04 \mathrm{~mm}$

2.07 to $24.85 \mathrm{deg}$.

$-37<=\mathrm{h}<=29, \quad-10<=\mathrm{k}<=10, \quad-23<=\mathrm{l}<=23$

$36204 / 3897[R($ int $)=0.0870]$

$98.9 \%$

Semi-empirical from equivalents

$\odot .9591$ and 0.6545

Full-matrix least-squares on $\mathrm{F}^{\wedge} 2$

$3897 / \odot / 299$

1.029

$\mathrm{R} 1=0.0375, \mathrm{WR} 2=0.0822$

$\mathrm{R} 1=0.0676, \mathrm{WR} 2=0.0942$

1.120 and -0.778 e. $A^{\wedge}-3$ 
Table 2. Atomic coordinates $(x 10 \wedge 4)$ and equivalent isotropic displacement parameters $\left(A^{\wedge} 2 \times 10 \wedge 3\right)$ for $m r 17$.

$\mathrm{U}(\mathrm{eq})$ is defined as one third of the trace of the orthogonalized Uij tensor.

\begin{tabular}{|c|c|c|c|c|}
\hline & $x$ & y & $z$ & $\mathrm{U}(\mathrm{eq})$ \\
\hline $\operatorname{Pd}(1)$ & $4624(1)$ & $1392(1)$ & $5686(1)$ & $24(1)$ \\
\hline $\mathrm{Cl}(1)$ & $5424(1)$ & $2630(1)$ & $6551(1)$ & $35(1)$ \\
\hline$S(1)$ & $3584(1)$ & $-126(1)$ & $5156(1)$ & $24(1)$ \\
\hline$N(1)$ & $4188(2)$ & $3276(4)$ & $5205(2)$ & $27(1)$ \\
\hline$N(2)$ & $3949(1)$ & $315(4)$ & $4869(2)$ & $22(1)$ \\
\hline$N(3)$ & $5008(1)$ & $-549(4)$ & $6099(2)$ & $24(1)$ \\
\hline $0(1)$ & $3924(1)$ & $-839(3)$ & $5969(2)$ & $30(1)$ \\
\hline $0(2)$ & $3125(1)$ & $-9 \odot \odot(3)$ & $4465(2)$ & $28(1)$ \\
\hline$C(1)$ & $4339(2)$ & $4378(5)$ & $5759(3)$ & $33(1)$ \\
\hline$C(2)$ & $4103(2)$ & $5759(5)$ & $5502(4)$ & $38(1)$ \\
\hline$c(3)$ & $3733(2)$ & $6016(5)$ & $4654(4)$ & $39(1)$ \\
\hline$C(4)$ & $3567(2)$ & $4901(5)$ & $4052(3)$ & $33(1)$ \\
\hline$c(5)$ & $3185(2)$ & $5135(6)$ & $3162(4)$ & $40(1)$ \\
\hline$c(6)$ & $3033(2)$ & $4054(6)$ & $2592(4)$ & $42(1)$ \\
\hline$C(7)$ & $3231(2)$ & $2605(6)$ & $2877(3)$ & $32(1)$ \\
\hline$C(8)$ & $3070(2)$ & $1466(6)$ & $2277(3)$ & 41(1) \\
\hline$C(9)$ & $3237(2)$ & $66(6)$ & $2528(3)$ & $40(1)$ \\
\hline$C(10)$ & $3530(2)$ & $-282(5)$ & $3396(3)$ & $31(1)$ \\
\hline$c(11)$ & $3683(2)$ & $769(5)$ & $400 \odot(3)$ & $26(1)$ \\
\hline$C(12)$ & $3581(2)$ & $2282(5)$ & $3758(3)$ & $29(1)$ \\
\hline$C(13)$ & $3787(2)$ & 3464 (5) & $4358(3)$ & $28(1)$ \\
\hline$c(14)$ & $3356(2)$ & $1506(5)$ & $5319(3)$ & $25(1)$ \\
\hline$C(15)$ & $2943(2)$ & $2282(5)$ & $4618(3)$ & $27(1)$ \\
\hline$C(16)$ & $2828(2)$ & $3686(5)$ & $4746(3)$ & $30(1)$ \\
\hline$c(17)$ & $3111(2)$ & $4313(5)$ & $5549(3)$ & $28(1)$ \\
\hline$C(18)$ & $3495(2)$ & $3479(5)$ & $6241(3)$ & $34(1)$ \\
\hline$C(19)$ & $3626(2)$ & $2095(5)$ & $6137(3)$ & $29(1)$ \\
\hline$c(20)$ & $2997(2)$ & $5868(5)$ & $5652(4)$ & $40(1)$ \\
\hline$C(21)$ & $4919(2)$ & $-1551(5)$ & $5524(3)$ & $26(1)$ \\
\hline$c(22)$ & $5167(2)$ & $-2898(5)$ & $5770(3)$ & $29(1)$ \\
\hline$c(23)$ & $5514(2)$ & $-3238(5)$ & $6617(3)$ & $31(1)$ \\
\hline$C(24)$ & $5605(2)$ & $-2212(5)$ & $7213(3)$ & $34(1)$ \\
\hline$C(25)$ & $5348(2)$ & $-895(5)$ & $6930(3)$ & $30(1)$ \\
\hline
\end{tabular}


Table 3. Bond lengths [A] and angles [deg] for mr17.

\begin{tabular}{|c|c|}
\hline$P d(1)-N(2)$ & $1.997(4)$ \\
\hline$P d(1)-N(3)$ & $2.026(4)$ \\
\hline$P d(1)-N(1)$ & $2.050(4)$ \\
\hline $\operatorname{Pd}(1)-\operatorname{Cl}(1)$ & $2.3277(12)$ \\
\hline$S(1)-0(1)$ & $1.437(3)$ \\
\hline$s(1)-0(2)$ & $1.444(3)$ \\
\hline $\mathrm{S}(1)-\mathrm{N}(2)$ & $1.617(4)$ \\
\hline$S(1)-C(14)$ & $1.769(5)$ \\
\hline$N(1)-C(1)$ & $1.343(6)$ \\
\hline$N(1)-C(13)$ & $1.366(6)$ \\
\hline$N(2)-C(11)$ & $1.438(6)$ \\
\hline$N(3)-C(25)$ & $1.345(6)$ \\
\hline$N(3)-C(21)$ & $1.347(6)$ \\
\hline$c(1)-c(2)$ & $1.398(7)$ \\
\hline$C(2)-c(3)$ & $1.362(8)$ \\
\hline$c(3)-c(4)$ & $1.403(7)$ \\
\hline$c(4)-c(5)$ & $1.422(7)$ \\
\hline$C(4)-C(13)$ & $1.439(6)$ \\
\hline$C(5)-C(6)$ & $1.347(8)$ \\
\hline$c(6)-c(7)$ & $1.429(7)$ \\
\hline$C(7)-C(12)$ & $1.416(7)$ \\
\hline$C(7)-C(8)$ & $1.417(7)$ \\
\hline$C(8)-c(9)$ & $1.361(7)$ \\
\hline$C(9)-C(10)$ & $1.402(7)$ \\
\hline$C(10)-C(11)$ & $1.369(6)$ \\
\hline$c(11)-c(12)$ & $1.435(7)$ \\
\hline$c(12)-c(13)$ & $1.435(7)$ \\
\hline$C(14)-C(15)$ & $1.393(6)$ \\
\hline$C(14)-C(19)$ & $1.397(6)$ \\
\hline$C(15)-C(16)$ & $1.398(6)$ \\
\hline$c(16)-c(17)$ & $1.387(7)$ \\
\hline$C(17)-C(18)$ & $1.386(7)$ \\
\hline$C(17)-C(20)$ & $1.510(7)$ \\
\hline$c(18)-c(19)$ & $1.385(7)$ \\
\hline$C(21)-C(22)$ & $1.382(6)$ \\
\hline$C(22)-c(23)$ & $1.369(7)$ \\
\hline$c(23)-c(24)$ & $1.389(7)$ \\
\hline$C(24)-C(25)$ & $1.371(7)$ \\
\hline$N(2)-P d(1)-N(3)$ & $89.14(14)$ \\
\hline$N(2)-P d(1)-N(1)$ & $86.84(14)$ \\
\hline$N(3)-P d(1)-N(1)$ & $175.97(15)$ \\
\hline$N(2)-P d(1)-C l(1)$ & $175.04(11)$ \\
\hline$N(3)-P d(1)-C l(1)$ & $90.30(11)$ \\
\hline$N(1)-P d(1)-C l(1)$ & $93.67(11)$ \\
\hline $0(1)-S(1)-0(2)$ & $118.70(19)$ \\
\hline $\mathrm{O}(1)-\mathrm{S}(1)-\mathrm{N}(2)$ & $106.6(2)$ \\
\hline$O(2)-S(1)-N(2)$ & $108.75(19)$ \\
\hline$O(1)-S(1)-C(14)$ & $107.3(2)$ \\
\hline$O(2)-S(1)-C(14)$ & $107.1(2)$ \\
\hline$N(2)-S(1)-C(14)$ & $108.0(2)$ \\
\hline$C(1)-N(1)-C(13)$ & $120.6(4)$ \\
\hline$C(1)-N(1)-P d(1)$ & $116.4(3)$ \\
\hline
\end{tabular}




\begin{tabular}{ll}
$C(13)-N(1)-P d(1)$ & $122.8(3)$ \\
$C(11)-N(2)-S(1)$ & $117.2(3)$ \\
$C(11)-N(2)-P d(1)$ & $113.0(3)$ \\
$S(1)-N(2)-P d(1)$ & $119.2(2)$ \\
$C(25)-N(3)-C(21)$ & $117.9(4)$ \\
$C(25)-N(3)-P d(1)$ & $122.8(3)$ \\
$C(21)-N(3)-P d(1)$ & $119.3(3)$ \\
$N(1)-C(1)-C(2)$ & $122.2(5)$ \\
$C(3)-C(2)-C(1)$ & $118.6(5)$ \\
$C(2)-C(3)-C(4)$ & $121.0(5)$ \\
$C(3)-C(4)-C(5)$ & $122.5(5)$ \\
$C(3)-C(4)-C(13)$ & $118.1(5)$ \\
$C(5)-C(4)-C(13)$ & $119.4(5)$ \\
$C(6)-C(5)-C(4)$ & $121.7(5)$ \\
$C(5)-C(6)-C(7)$ & $120.2(5)$ \\
$C(12)-C(7)-C(8)$ & $119.3(5)$ \\
$C(12)-C(7)-C(6)$ & $120.6(5)$ \\
$C(8)-C(7)-C(6)$ & $120.2(5)$ \\
$C(9)-C(8)-C(7)$ & $121.6(5)$ \\
$C(8)-C(9)-C(10)$ & $118.9(5)$ \\
$C(11)-C(10)-C(9)$ & $121.6(5)$ \\
$C(10)-C(11)-C(12)$ & $119.9(4)$ \\
$C(10)-C(11)-N(2)$ & $118.4(4)$ \\
$C(12)-C(11)-N(2)$ & $121.7(4)$ \\
$C(7)-C(12)-C(13)$ & $118.9(4)$ \\
$C(7)-C(12)-C(11)$ & $117.4(5)$ \\
$C(13)-C(12)-C(11)$ & $123.6(4)$ \\
$N(1)-C(13)-C(12)$ & $122.4(4)$ \\
$N(1)-C(13)-C(4)$ & $119.1(4)$ \\
$C(12)-C(13)-C(4)$ & $118.5(4)$ \\
$C(15)-C(14)-C(19)$ & $120.0(4)$ \\
$C(15)-C(14)-S(1)$ & $119.6(3)$ \\
$C(19)-C(14)-S(1)$ & $119.9(3)$ \\
$C(14)-C(15)-C(16)$ & $118.8(4)$ \\
$C(17)-C(16)-C(15)$ & $121.6(4)$ \\
$C(18)-C(17)-C(16)$ & $118.2(4)$ \\
$C(18)-C(17)-C(20)$ & $121.8(5)$ \\
$C(16)-C(17)-C(20)$ & $120.0(5)$ \\
$C(19)-C(18)-C(17)$ & $121.6(5)$ \\
$C(18)-C(19)-C(14)$ & $119.5(4)$ \\
$N(3)-C(21)-C(22)$ & $121.7(4)$ \\
$C(23)-C(22)-C(21)$ & $119.9(5)$ \\
$C(22)-C(23)-C(24)$ & $118.6(4)$ \\
$C(25)-C(24)-C(23)$ & $118.7(4)$ \\
$N(3)-C(25)-C(24)$ & $123.1(5)$ \\
\hline & \\
\hline &
\end{tabular}

Symmetry transformations used to generate equivalent atoms: 
Table 4. Anisotropic displacement parameters $\left(A^{\wedge} 2 \times 10 \wedge 3\right)$ for $m r 17$. The anisotropic displacement factor exponent takes the form:

-2 pi^2 [ h^2 $\left.a^{* \wedge 2} \mathrm{U} 11+\ldots+2 \mathrm{~h} k \mathrm{a}^{*} \mathrm{~b}^{*} \mathrm{U} 12\right]$

\begin{tabular}{|c|c|c|c|c|c|c|}
\hline & U11 & U22 & U33 & U23 & U13 & U12 \\
\hline $\mathrm{Pd}(1)$ & $23(1)$ & $16(1)$ & $24(1)$ & $-3(1)$ & $10(1)$ & $\Theta(1)$ \\
\hline $\mathrm{Cl}(1)$ & $30(1)$ & $27(1)$ & $31(1)$ & $-7(1)$ & $10(1)$ & $-5(1)$ \\
\hline$S(1)$ & $27(1)$ & $16(1)$ & $26(1)$ & $\odot(1)$ & $14(1)$ & $1(1)$ \\
\hline$N(1)$ & $27(2)$ & $20(2)$ & $32(2)$ & $-4(2)$ & $17(2)$ & $-4(2)$ \\
\hline$N(2)$ & $25(2)$ & $13(2)$ & $24(2)$ & $3(2)$ & $14(2)$ & $6(2)$ \\
\hline$N(3)$ & $19(2)$ & $24(2)$ & $22(2)$ & $-2(2)$ & $9(2)$ & $\odot(2)$ \\
\hline $0(1)$ & $39(2)$ & $21(2)$ & $26(2)$ & $6(1)$ & $17(2)$ & $6(2)$ \\
\hline $0(2)$ & $24(2)$ & $19(2)$ & $32(2)$ & $-5(1)$ & $12(2)$ & $-3(1)$ \\
\hline$C(1)$ & $35(3)$ & $29(3)$ & $37(3)$ & $-6(2)$ & $22(3)$ & $-4(2)$ \\
\hline$C(2)$ & $46(3)$ & $16(3)$ & $64(4)$ & $-3(3)$ & $39(3)$ & $-2(2)$ \\
\hline$c(3)$ & $47(3)$ & $15(3)$ & $66(4)$ & $4(3)$ & $40(3)$ & $2(2)$ \\
\hline$C(4)$ & $34(3)$ & $18(3)$ & $53(3)$ & $13(2)$ & $29(3)$ & $6(2)$ \\
\hline$C(5)$ & $36(3)$ & $29(3)$ & $52(4)$ & $17(3)$ & $25(3)$ & $11(2)$ \\
\hline$c(6)$ & $35(3)$ & $41(3)$ & $38(3)$ & $11(3)$ & $15(3)$ & $2(3)$ \\
\hline$C(7)$ & $25(3)$ & $40(3)$ & $26(3)$ & $6(2)$ & $12(2)$ & $-3(2)$ \\
\hline$C(8)$ & $39(3)$ & $46(3)$ & $22(3)$ & $3(3)$ & $11(2)$ & $-8(3)$ \\
\hline$C(9)$ & $44(3)$ & $35(3)$ & $28(3)$ & $-7(2)$ & $16(3)$ & $-9(3)$ \\
\hline$C(10)$ & $31(3)$ & $26(3)$ & $28(3)$ & $-1(2)$ & $14(2)$ & $-2(2)$ \\
\hline$C(11)$ & $17(2)$ & $25(3)$ & $29(3)$ & $1(2)$ & $11(2)$ & $-1(2)$ \\
\hline$C(12)$ & $24(3)$ & $30(3)$ & $30(3)$ & $6(2)$ & $15(2)$ & $5(2)$ \\
\hline$C(13)$ & $25(3)$ & $24(3)$ & $37(3)$ & $5(2)$ & $20(2)$ & $2(2)$ \\
\hline$C(14)$ & $23(2)$ & $19(2)$ & $29(3)$ & $\odot(2)$ & $14(2)$ & $\odot(2)$ \\
\hline$C(15)$ & $24(3)$ & $29(3)$ & $23(3)$ & $2(2)$ & $11(2)$ & $1(2)$ \\
\hline$C(16)$ & $27(3)$ & $28(3)$ & $30(3)$ & $11(2)$ & $16(2)$ & $6(2)$ \\
\hline$C(17)$ & $29(3)$ & $23(3)$ & $38(3)$ & $1(2)$ & $24(3)$ & $1(2)$ \\
\hline$C(18)$ & $33(3)$ & $35(3)$ & $33(3)$ & $-5(2)$ & $20(3)$ & $\odot(2)$ \\
\hline$C(19)$ & $25(3)$ & $27(3)$ & $23(3)$ & $1(2)$ & $8(2)$ & $2(2)$ \\
\hline$C(20)$ & $49(4)$ & $25(3)$ & $57(4)$ & $-2(3)$ & $37(3)$ & $2(3)$ \\
\hline$C(21)$ & $25(3)$ & $23(3)$ & $26(3)$ & $1(2)$ & $14(2)$ & $-2(2)$ \\
\hline$C(22)$ & $28(3)$ & $23(3)$ & $34(3)$ & $-4(2)$ & $18(2)$ & $-1(2)$ \\
\hline$C(23)$ & $25(3)$ & $21(3)$ & $43(3)$ & $4(2)$ & $17(3)$ & $3(2)$ \\
\hline$C(24)$ & $31(3)$ & $35(3)$ & $25(3)$ & $8(2)$ & $12(2)$ & $5(2)$ \\
\hline$C(25)$ & $26(3)$ & $28(3)$ & $27(3)$ & $-3(2)$ & $11(2)$ & $\odot(2)$ \\
\hline
\end{tabular}


Table 5. Hydrogen coordinates $(x 10 \wedge 4)$ and isotropic displacement parameters $\left(A^{\wedge} 2 \times 10 \wedge 3\right)$ for $m r 17$.

\begin{tabular}{lcccc}
\hline & $x$ & $y$ & $z$ & $U(e q)$ \\
\hline$H(1 B)$ & 4615 & 4214 & 6345 & 40 \\
$H(2 B)$ & 4200 & 6502 & 5909 & 46 \\
$H(3 A)$ & 3584 & 6965 & 4467 & 47 \\
$H(5 A)$ & 3032 & 6078 & 2964 & 48 \\
$H(6 A)$ & 2794 & 4256 & 1999 & 50 \\
$H(8 A)$ & 2840 & 1687 & 1686 & 49 \\
$H(9 A)$ & 3157 & -664 & 2122 & 47 \\
$H(10 A)$ & 3624 & -1272 & 3570 & 37 \\
$H(15 A)$ & 2744 & 1865 & 4064 & 33 \\
$H(16 A)$ & 2550 & 4223 & 4270 & 36 \\
$H(18 A)$ & 3674 & 3865 & 6799 & 40 \\
$H(19 A)$ & 3897 & 1550 & 6618 & 35 \\
$H(20 A)$ & 3037 & 6517 & 5297 & 60 \\
$H(20 B)$ & 3246 & 6161 & 6250 & 60 \\
$H(20 C)$ & 2636 & 5934 & 5475 & 60 \\
$H(21 A)$ & 4681 & -1326 & 4934 & 31 \\
$H(22 A)$ & 5097 & -3586 & 5351 & 34 \\
$H(23 A)$ & 5689 & -4157 & 6793 & 38 \\
$H(24 A)$ & 5841 & -2420 & 7805 & 40 \\
$H(25 A)$ & 5413 & -194 & 7340 & 36 \\
\hline
\end{tabular}

References:

${ }^{1}$ Yamada, Y.; Yamamoto, T.; Okawara, M. Chem. Lett. 1975, 361-362.

2 Södergren, M. J.; Alonso, D. A.; Bedekar, A. V.; Andersson, P. G. Tetrahedron Lett. 1997, 38, 6897-6900.

${ }^{3}$ Cope, A. C.; Siekman, R. W. J. Am. Chem. Soc. 1965, 87, 3272-3273.

${ }^{4}$ Nonoyama, M.; Suzuki, K; Yamasaki, K. Proc. Japan Acad. 1969, 45, 605-608.

${ }^{5}$ Holcomb, H. L.; Nakanishi, S.; Flood, T. C. Organometallics 1996, 15, 4228-4234. 